\title{
Root Systems and Periods on Hirzebruch Surfaces
}

\author{
By \\ Jun-ichi MatsuzawA*
}

\section{§ I. Introduction}

Cayley classified all nonsingular cubic surfaces in three-dimensional complex projective space by using the configuration of the 27 lines on the surface [3]. The symmetry of these 27 lines can be described by the Weyl group and root system of type $E_{6}([4])$. Furthermore these objects, namely the 27 lines, the Weyl group of type $E_{6}$, and root system of type $E_{6}$, have natural realization in the Picard group of cubic surface ([8]).

In [10] a fine moduli space $M$ of marked cubic surfaces was constructed explicitly in such a manner that the relation between the geometrical structure of $M$ and the structure of root system became clear. On the other hand the fine moduli spaces for certain classes of rational surfaces were constructed in terms of root system and periods, which are integrals of a meromorphic 2-form over 2-cycles on the surface corresponding to roots ([7]). The moduli space $M$ was reconstructed in terms of the root system and the periods in the same way (Appendix in [10]).

In this paper, we discuss the moduli problem for certain class of rational surfaces in terms of the root system of type $A$. Let $X$ be the rational surface obtained from the $n$-th Hirzebruch surface or rational ruled surface with in variant $n$ by blowing up $n$ points. The relation between the Hirzebruch surfaces with $n$ points blown up and the root system of type $A_{n-1}$ is similar to the relation between the cubic surface and the root system of type $E_{6}$. We prove a Torelli theorem for the pairs of $X$ and a certain anticanonical divisor on $X$ by using the structure of the root system of type $A_{n-1}$ in the Picard group of $X$.

We shall construct a family $p: \mathfrak{X} \rightarrow S$ of the Hirzebruch surfaces with $n$ points blown up and study a period mapping for the fibration $p: \mathfrak{X} \rightarrow S$, where the base space $S$ is the quotient space of a maximal torus of the simple Lie group of type $A_{n-1}$ by its Weyl group. The fiber $\mathfrak{x}_{t}$ of $p$ can be regarded as a compactification of the fiber of semi-universal deformation of the simple sur-

Communicated by K. Saito, April 9, 1992.

1991 Mathematics Subject Classifications : 14J10 (14J26)

* Department of Mathematics, Faculty of Science, Kyoto Lniversity, Kyoto 606, Japan. 
face singularity of type $1_{n-1}$. This relation between the Hirzebruch surfaces with $n$ points blown up and simple surface singularities of type $A_{n-1}$ is similar to the relation between Del Pezzo surfaces and simple surface singularities of type $E$ (see Remark 5.4). In order to define a period mapping, we fix a meromorphic 2 -form $\omega$ on $\mathfrak{x}$. Denote by $\Delta \subset S$ the discriminant variety of $p$ and by $\mathfrak{D}_{t}(t \in S \backslash \Delta)$ the anticanonical divisor on the fiber $\mathfrak{x}_{t}$ such that the restriction of $\omega$ to the fiber $\mathfrak{x}_{t}$ has poles only along $\mathfrak{D}_{t}$. The fundamental group $\pi_{1}(S \backslash \Delta)$ of the space $S \backslash \Delta$ is isomorphic to the Artin group associated to the extended Dynkin diagram of type $A_{n-1}([11])$. The monodromy group or the image of the monodromy representation of $\pi_{1}(S \backslash \Delta)$ on the second homology group of $\ddot{x}_{t} \backslash \mathscr{D}_{t}$ is isomorphic to the affine Weyl group $\widetilde{W}$ of the root system of type $A_{n-1}$. The group $\pi_{1}(S \backslash \Delta)$ acts on the period domain as an affine transformation group which is isomorphic to $\widetilde{W}$.

It is a pleasure to acknowledge many helpful discussions with Ikuo Satake about the work in this paper. I would also like to thank K. Irie for calculating the ranks of the homology groups of $\ddot{x}_{t} \backslash \mathfrak{D}_{t}$ and for helpful conversation about questions in topology. I finally express my gratitude to I. Naruki and K. Saito for giving me many valuable suggestions.

\section{$\S 2$. Hirzebruch Surfaces with Several Points Blown up}

We denote by $\Sigma_{n}, n \geqq 0$, the $n$-th Hirzebruch surface or the rational ruled surface with invariant $n$. The surface $\Sigma_{n}$ is a $\boldsymbol{P}^{1}$-bundle over $\boldsymbol{P}^{1}$. For $n \geqq 1$, $\Sigma_{n}$ are obtained by desingularising the projective cone $Y$ in $\boldsymbol{P}^{n+1}$ over a nonsingular rational curve of degree $n$ which lies in a hyperplane of $P^{n+1}$. Especially $\Sigma_{0}$ is $\mathbb{P}^{1} \times \mathbb{P}^{1}$. The Hirzebruch surfaces have only one ruling except for $\Sigma_{0}$ (see e. g. [1, Chap. V. 4], [6, Chap. V]). For $n \geqq 1$, let $\pi: \Sigma_{n} \rightarrow \mathbb{P}^{1}$ be the ruling and $S$ the unique section with $S \cdot S=-n$.

For later use, we shall give another realization of $\Sigma_{n}$. $\Sigma_{n}$ is isumorphic to the variety in $\boldsymbol{P}^{2} \times \boldsymbol{P}^{1}$

$$
\Sigma_{n}=\left\{\left(\zeta_{0}: \zeta_{1}: \zeta_{2}\right)(s: t) \in \boldsymbol{P}^{2} \times \boldsymbol{P}^{1} \mid s^{n} \zeta_{0}=t^{n} \zeta_{1}\right\} .
$$

The second projection gives the ruling and

$$
S=\left\{\left(\zeta_{0}: \zeta_{1}: \zeta_{2}\right)(s: t) \equiv \Sigma_{n} \mid \zeta_{0}=\zeta_{1}=0\right\}
$$

Put

$$
\left\{\begin{array}{l}
F=\left\{\left(\zeta_{0}: \zeta_{1}: \zeta_{2}\right)(s: t) \subseteq \Sigma_{n} \mid s=0\right\}, \\
F^{\prime}=\left\{\left(\zeta_{0}: \zeta_{1}: \zeta_{2}\right)(s: t) \equiv \Sigma_{n} \mid t=0\right\}, \\
C_{0}=\left\{\left(\zeta_{0}: \zeta_{1}: \zeta_{2}\right)(s: t) \subseteq \Sigma_{n} \mid \zeta_{2}=0\right\}
\end{array}\right.
$$

Then $F$ and $F^{\prime}$ are two fibers and $C_{0}$ is a section with $C_{0} \cdot C_{0}=n . \quad \Sigma_{n}$ is 
covered by 4 copies $U_{\imath}, 1 \leqq i \leqq 4$, of $C^{2}$ with coordinates $\left(z_{1}^{(i)}, z_{2}^{(i)}\right)$, which are defined by

$$
\begin{cases}U_{1}=\Sigma_{n} \backslash\left(F^{\prime} \cup S\right), & \left(z_{1}^{(1)}, z_{2}^{(1)}\right)=\left(s / t, \zeta_{2} / \zeta_{0}\right), \\ U_{2}=\Sigma_{n} \backslash(F \cup S), & \left(z_{1}^{(2)}, z_{2}^{(2)}\right)=\left(t / s, \zeta_{2} / \zeta_{1}\right), \\ U_{3}=\Sigma_{n} \backslash\left(F \cup C_{0}\right), & \left(z_{1}^{(3)}, z_{2}^{(3)}\right)=\left(t / s, \zeta_{1} / \zeta_{2}\right), \\ U_{4}=\Sigma_{n} \backslash\left(F^{\prime} \cup C_{0}\right), & \left(z_{1}^{(4)}, z_{2}^{(4)}\right)=\left(s / t, \zeta_{0} / \zeta_{2}\right) .\end{cases}
$$

The transition functions among these coordinates are given by

$$
\left\{\begin{array}{l}
z_{1}^{(1)} z_{1}^{(2)}=z_{1}^{(2)} z_{1}^{(4)}=1, \quad z_{1}^{(2)}=z_{1}^{(3)} \\
z_{2}^{(2)}=\left(z_{1}^{(1)}\right)^{-n} z_{2}^{(1)}, \quad z_{2}^{(1)} z_{2}^{(4)}=1, \quad z_{2}^{(2)} z_{2}^{(3)}=1 .
\end{array}\right.
$$

Definition 2.1. We say that $n$ points $P_{1}, \cdots, P_{n}$ of $\Sigma_{n}$ are 'in general position' if no two of them lie on a fiber and no one lies on the section $S$.

Remark 2.2. For $n \geqq 1$, let $\Phi: \Sigma_{n} \rightarrow Y$ be the morphism obtained by desingularising the projective cone $Y$ in $P^{n+1}$. If $n$ points $P_{1}, \cdots, P_{n}$ of $\Sigma_{n}$ are in general position, the $n$ points $Q_{\imath}=\Phi\left(P_{i}\right), 1 \leqq i \leqq n$, are contained in a unique hyperplane $H$ such that $H$ does not pass the verlex of $Y$ and the intersection $H \cap Y$ is an irreducible nonsingular curve. By Bertini's theorem, the set of hypersurfaces $H$ such that $H \cap Y$ is an irreducible nonsingular curve is an open dense subset of the complete linear system $|H|$, considered as a projective space. Therefore the set of the points $\left(P_{1}, \cdots, P_{n}\right)$ in general position is an open dense subset of the variety $\Sigma_{n} \times \cdots \times \Sigma_{n}$ ( $n$ times).

From now on, we assume that $n \geqq 1$. Let $P_{1}, \cdots, P_{n}$ be $n$ points of $\Sigma_{n}$ in general position and

$$
p: X_{n} \longrightarrow \Sigma_{n}
$$

the morphism obtained by blowing up these $n$ points.

Let $f$ and $s$ be the linear equivalence classes of the total transforms of a fiber of $\pi$ and the section $S$ respectively. Let $E_{1}, \cdots, E_{n}\left(E_{i}=\pi^{-1}\left(P_{\iota}\right)\right)$ be the exceptional curves and $e_{1}, \cdots, e_{n}$ the linear equivalence classes of them. Then we have (see e.g. [6, Chap. V])

\section{Proposition 2.3.}

(1) The Picard group Pic $\left(X_{n}\right)$ is generated by $f, s, e_{1}, \cdots, e_{n}$.

(2) The intersection pairing on $X_{n}$ is given by $f^{2}=0, s^{2}=-n, e_{1}^{2}=-1$ $(1 \leqq i \leqq n), f \cdot s=1, f \cdot e_{\imath}=0, s \cdot e_{\imath}=0, e_{i} \cdot e_{j}=0(i \neq j)$.

(3) The canonical class is $k=-(n+2) f-2 s+e_{1}+\cdots+e_{n}$.

(4) Let $C$ be an irreducible curve on $X_{n}$, other than $E_{1}, \cdots, E_{n}$, and $c=x f$ $+y s-\sum_{i=1}^{n} b_{\imath} e_{i}$ the linear equivalence class of $C$. Then we have either (i) $x=1$, 
$y=0, b_{i} \geqq 0(1 \leqq i \leqq n)$, (ii) $x=0, y=1, b_{i}=0(1 \leqq i \leqq n)$, or (iii) $x \geqq n y, y>0, b_{i} \geqq 0$ $(1 \leqq i \leqq n)$.

Notation 2.4. Let $D$ be an anticanonical divisor on $X_{n}$ whose irreducible components are the proper transforms $\bar{F}, \bar{F}^{\prime}$ of two distinct fibers $F, F^{\prime}$ of the projection $\pi: \Sigma_{n} \rightarrow \boldsymbol{P}^{1}$ not containing $P_{1}, \cdots, P_{n}$, the proper transform $\bar{S}$ of the section $S$, and the proper transform $\bar{C}$ of the section $C$ of $\pi$ with $C^{2}=n$ which passes through $P_{1}, \cdots, P_{n}$. We shall give an order $\bar{F}_{1}, \bar{F}_{2}$ to these two components $\bar{F}, \bar{F}^{\prime}$ and call it an orientation of the divisor

$$
D=\bar{F}_{1}+\bar{F}_{2}+\bar{S}+\bar{C} \text {. }
$$

By $\delta\left(X_{n}\right)$ we denote the set of such anticanonical divisors. Since the linear equivalence classes of $\bar{F}_{1}, \bar{F}_{2}, \bar{S}, \bar{C}$ are $f, f, s$, and $n f+s-e_{1}-\cdots-e_{n}$ respectively, the linear equivalence class of $D$ is $(n+2) f+2 s-e_{1}-\cdots-e_{n}$. Thus $D$ is an anticanonical divisor on $X_{n}$.

Definition 2.5. Let $X_{n}$ and $X_{n}^{\prime}$ be surfaces obtained by blowing up $n$ points in general position of $\Sigma_{n}$. For $D=\bar{F}_{1}+\bar{F}_{2}+\bar{S}+\bar{C} \Subset \mathfrak{D}\left(X_{n}\right)$ and $D^{\prime}=$ $\bar{F}_{1}^{\prime}+\bar{F}_{2}^{\prime}+\bar{S}^{\prime}+\bar{C}^{\prime} \in \mathfrak{D}\left(X_{n}^{\prime}\right)$, if there exists an isomorphism $\phi: X_{n} \rightarrow X_{n}^{\prime}$ such that

$$
\phi\left(\bar{F}_{i}\right)=\bar{F}_{i}^{\prime}(i=1,2), \quad \phi(\bar{S})=\bar{S}^{\prime}, \quad \phi(\bar{C})=\bar{C}^{\prime},
$$

then we say that the pairs $\left(X_{n}, D\right)$ and $\left(X_{n}^{\prime}, D^{\prime}\right)$ are isomorphic.

We next consider the isomorphic classes of the pairs $\left(X_{n}, D\right)$.

Lemma 2.6. Let $F_{0}=\pi^{-1}(0)$ and $F_{\infty}=\pi^{-1}(\infty)$ be fibers of $\Sigma_{n}$, $S$ the $(-n)$ section, and $C$ an n-section of $\pi: \Sigma_{n} \rightarrow \boldsymbol{P}^{1}$. Let $p^{\prime}: X_{n}^{\prime} \rightarrow \Sigma_{n}$ be a blowing-up at $n$ points in general position and $D^{\prime}=\bar{F}_{1}^{\prime}+\bar{F}_{2}^{\prime}+\bar{S}^{\prime}+\bar{C}^{\prime} \in \mathfrak{D}\left(X_{n}^{\prime}\right)$. Then there exists $n$ points $P_{1}, \cdots, P_{n}$ of $\Sigma_{n}$ in general position which have the following properties:

(1) $P_{1}, \cdots, P_{n} \equiv C \backslash\left(F_{0} \cup F_{\infty}\right)$,

(2) Let $X_{n}$ be the surface obtained by blowing up $P_{1}, \cdots, P_{n}$. Then there exists an isomorphism $\Phi: X_{n} \rightarrow Y_{n}^{\prime}$ such that

$$
\Phi\left(\bar{F}_{0}\right)=\bar{F}_{1}^{\prime}, \quad \Phi\left(\bar{F}_{\infty}\right)=\bar{F}_{2}^{\prime}, \quad \Phi(\bar{S})=\bar{S}^{\prime}, \quad \Phi(\bar{C})=\bar{C}^{\prime},
$$

where $\bar{F}_{0}, \bar{F}_{\infty}, \bar{S}, \bar{C}$ are the proper transforms of $F_{0}, F_{\infty}, S, C$ respectively.

Proof. The Hirzebruch surface $\Sigma_{n}$ can be obtained by blowing up the vertex $Q$ of a projective cone $Y$ in $\boldsymbol{P}^{n+1}$ over a nonsingular rational curve of degree $n$ lying in a hyperplane of $\boldsymbol{P}^{n+1}$. The $n$-sections of $\Sigma_{n}$ are the strict transforms of the hyperplane sections of $Y$ not containing the vertex $Q$. Let $H$ and $H^{\prime}$ be the hyperplanes in $P^{n+1}$ corresponding to $C$ and $C^{\prime}=p^{\prime}\left(\bar{C}^{\prime}\right)$. There exists a projective automorphism $\phi$ of $\boldsymbol{P}^{n+1}$ which sends $H$ to $H^{\prime}$ and preserves the cone $Y$. Let $\varphi_{1}$ be the automorphism of $\Sigma_{n}$ induced by $\phi$, which 
sends $C$ to $C^{\prime}$ and preserves the fibers. Let $\varphi_{2}$ be the automorphism of $\Sigma_{n}$ induced by the automorphism of $\pi\left(\Sigma_{n}\right) \cong \boldsymbol{P}^{1}$ which sends $F_{0}$ to $F_{1}^{\prime}=p^{\prime}\left(\bar{F}_{1}^{\prime}\right)$ and $F_{\infty}$ to $F_{2}^{\prime}=p^{\prime}\left(\bar{F}_{2}^{\prime}\right)$. Put $\varphi=\varphi_{1} \circ \varphi_{2}$. Then we have

$$
\varphi\left(F_{0}\right)=F_{1}^{\prime}, \quad \varphi\left(F_{\infty}\right)=F_{2}^{\prime}, \quad \varphi(S)=S^{\prime}, \quad \varphi(C)=C^{\prime} .
$$

Let $Q_{1}, \cdots, Q_{n}$ be the centers of the blowing-up $p^{\prime}: X_{n}^{\prime} \rightarrow \Sigma_{n}$. Put $P_{i}=\varphi^{-1}\left(Q_{i}\right)$, $1 \leqq i \leqq n$, then $P_{1}, \cdots, P_{n} \leqq C \backslash\left(F_{0} \cup F_{\infty}\right)$. Let $X_{n}$ be the surface obtained by blowing up $P_{1}, \cdots, P_{n}$ and $\Phi: X_{n} \rightarrow X_{n}^{\prime}$ the induced isomorphism by $\varphi$. Then $\Phi$ satisfies the second condition.

Proposition 2.7. Let $p: Y_{n} \rightarrow \Sigma_{n}$ (resp. $p^{\prime}: X_{n}^{\prime} \rightarrow \Sigma_{n}$ ) be the morphism obtained by blowing up $n$ points $P_{1}, \cdots, P_{n}$ (resp. $\left.P_{1}^{\prime}, \cdots, P_{n}^{\prime}\right)$ in general position and $D=$ $\bar{F}_{1}+\bar{F}_{2}+\bar{S}+\bar{C} \in \mathcal{D}\left(X_{n}\right)\left(\right.$ resp. $\left.D^{\prime}=\bar{F}_{1}^{\prime}+\bar{F}_{2}^{\prime}+\bar{S}^{\prime}+\bar{C}^{\prime} \boxminus \mathfrak{D}\left(X_{n}^{\prime}\right)\right)$. Let

$$
\begin{aligned}
& t_{0}=\pi\left(p\left(\bar{F}_{1}\right)\right), \quad t_{\infty}=\pi\left(p\left(\bar{F}_{2}\right)\right), \quad t_{i}=\pi\left(P_{i}\right) \cong \boldsymbol{P}^{1}, \quad 1 \leqq i \leqq n, \\
& t_{0}^{\prime}=\pi\left(p^{\prime}\left(\bar{F}_{1}^{\prime}\right)\right), \quad t_{\infty}^{\prime}=\pi\left(p^{\prime}\left(\bar{F}_{2}^{\prime}\right)\right), \quad t_{i}^{\prime}=\pi\left(P_{i}^{\prime}\right) \Subset \boldsymbol{P}^{1}, \quad 1 \leqq i \leqq n .
\end{aligned}
$$

Then the pairs $\left(Y_{n}, D\right)$ and $\left(X_{n}^{\prime}, D^{\prime}\right)$ are isomorphic if and only if there exists an automorphism $g$ of $\boldsymbol{P}^{1}$ such that

$$
\begin{gathered}
g\left(t_{0}\right)=t_{0}^{\prime}, \quad g\left(t_{\infty}\right)=t_{\infty}^{\prime}, \\
\left\{g\left(t_{1}\right), \cdots, g\left(t_{n}\right)\right\}=\left\{t_{1}^{\prime}, \cdots, t_{n}^{\prime}\right\} .
\end{gathered}
$$

Proof. This follows from Lemma 2.6.

\section{§3. Homology and Root System}

Throughout this section, we assume that $n \geqq 2$. We shall study the homology groups of the surfaces $X_{n}$ and $X_{n} \backslash D\left(D=\bar{F}_{1}+\bar{F}_{2}+\bar{S}+\bar{C} \subseteq \mathbb{D}\left(X_{n}\right)\right)$ with integral coefficients. The root systems of type $A_{n-1}$ can be realized in the homology groups of $X_{n}$. The realization is similar to that of the root systems in the homology groups of Del Pezzo surfaces and certain rational surfaces ([8], [7]).

We consider the homology exact sequence:

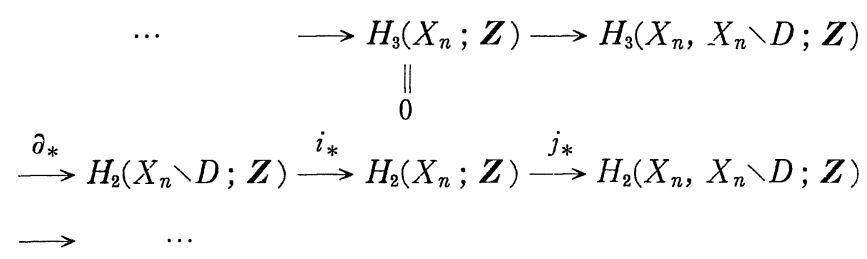

We extend the intersection form in $H_{2}\left(X_{n} ; \boldsymbol{Z}\right)$ to $H_{2}\left(X_{n} ; \boldsymbol{Z}\right) \otimes_{\boldsymbol{Z}} \boldsymbol{R}$. Let

$$
Q=\operatorname{ker} j_{*} \subset H_{2}\left(X_{n} ; \boldsymbol{Z}\right)
$$


and

$$
R=\{\alpha \in Q ; \alpha \cdot \alpha=-2\}
$$

Proposition 3.1. Let $Q$ and $R$ be as above. Then $Q$ is given by

$$
Q=\left\{\alpha \sqsubseteq H_{2}\left(X_{n} ; \boldsymbol{Z}\right) \mid \alpha \cdot f=\alpha \cdot s=\alpha \cdot\left(n f+s-e_{1}-\cdots-e_{n}\right)=0\right\}
$$

and $R$ is a root system of type $A_{n-1}$ in $Q \otimes_{\mathbf{Z}} \boldsymbol{R}$ and $Q$ is generated by $R$. The set $\Pi=\left\{e_{i}-e_{i+1} \mid 1 \leqq i \leqq n\right\}$ is a basis of $R$, where $e_{i}$ is the class of the exceptional curve $E_{i}=p^{-1}\left(P_{i}\right)$.

Proof. We have the following duality:

$$
H_{2}\left(X_{n}, X_{n} \backslash D ; Z\right) \cong H^{2}(D ; Z)
$$

Thus $\operatorname{ker} j_{*}$ is the lattice whose elements are orthogonal to the classes of the components of $D$. Since the classes of the components of $D$ are $f, s$, and $n f+s-e_{1}-\cdots-e_{n}$ (see Notation 2.4), we have (3.1).

Let $\alpha=x f+y s+\sum_{i=1}^{n} b_{i} e_{i}$ be an element of $Q$. It follows from

that

$$
\alpha \cdot f=\alpha \cdot s=\alpha \cdot\left(n f+s-e_{1}-\cdots-e_{n}\right)=0
$$

$$
x=y=0, \quad \sum_{i=1}^{n} b_{i}=0 .
$$

Thus

$$
Q=\left\{\sum_{i=1}^{n} b_{i} e_{i} \in H_{2}\left(X_{n} ; Z\right) \mid \sum_{i=1}^{n} b_{i}=0\right\} .
$$

Let $\alpha=\sum_{i=1}^{n} b_{i} e_{i} \in R$. Since $\alpha \cdot \alpha=-2$, we have $\sum_{i=1}^{n} b_{i}^{2}=2$. Thus $b_{i}= \pm 1, b_{j}=$ \pm 1 for some $i, j(i \neq j)$ and the rest are 0. By (3.2), $\alpha$ must be $\pm\left(e_{i}-e_{j}\right)$ and we have $R=\left\{e_{i}-e_{j} \mid i \neq j\right\}$. Therefore $Q$ is generated by $R$. Furthermore $R$ is a root system of type $A_{n-1}$ in $Q \otimes_{\boldsymbol{Z}} \boldsymbol{R}$ and $\Pi$ is a basis of $R$.

We next consider the second homology group $H_{2}\left(X_{n} \backslash D ; \boldsymbol{Z}\right)$. By Proposition 3.1 , we have the short exact sequence

$$
0 \longrightarrow H_{3}\left(X_{n}, X_{n} \backslash D ; \boldsymbol{Z}\right) \stackrel{\partial_{*}}{\longrightarrow} H_{2}\left(X_{n} \backslash D ; \boldsymbol{Z}\right) \stackrel{i_{*}}{\longrightarrow} Q \longrightarrow 0 .
$$

Let $E_{i}=p^{-1}\left(P_{i}\right)$ and $E_{j}=p^{-1}\left(P_{j}\right)$ be the exceptional curves and $B_{i}=E_{i} \cap \bar{C}$. Let $T$ be a closed tubular neighborhood of $\bar{C}$ in $X_{n}$ such that $T \cap E_{i}$ and $T \cap E_{j}$ are fibers. Let $\gamma$ be an injective path in $\bar{C}$ from $B_{i}$ to $B_{j}$ and let

$$
\Gamma_{i, j}=\left.\left(E_{i} \backslash\left(E_{\imath} \cap T\right)\right) \cup \partial T\right|_{r} \cup\left(E_{j} \backslash\left(E_{j} \cap T\right)\right) .
$$

We can take the orientation such that $\Gamma_{i, j}$ is homologous to $E_{i}-E_{j}$ in $X_{n}$. Hence we have 


$$
i_{*}\left(\left[\Gamma_{i, j}\right]\right)=e_{\imath}-e_{j} .
$$

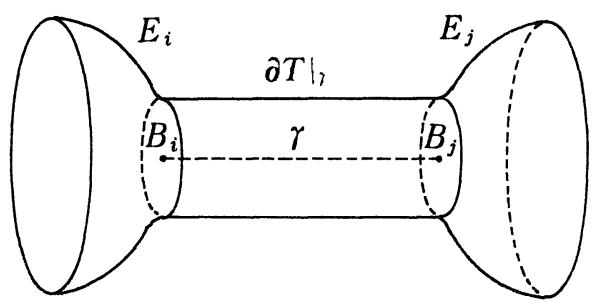

We shall write

$$
D=D_{1}+D_{2}+D_{3}+D_{4},
$$

where $D_{1}=\bar{F}_{1}, D_{2}=\bar{C}, D_{3}=\bar{F}_{2}$, and $D_{4}=\bar{S}$.

By Lemma 2.6 and (2.4), we can take a local coordinate $\left(z_{1}^{(2)}, z_{2}^{(i)}\right)(1 \leqq i \leqq 4)$ around the intersection $D_{\imath} \cap D_{\imath+1}$ on $X_{n}\left(D_{5}=D_{1}\right)$. For $r_{1}^{(\imath)}, r_{2}^{(i)}>0$, let $N_{\imath}$ $(1 \leqq i \leqq 4)$ be the 2 -cycle on $X_{n} \backslash D$ defined by

$$
N_{\imath}=\left\{\left(z_{1}^{(i)}, z_{2}^{(i)}\right)|| z_{1}^{(i)}\left|=r_{1}^{(i)},\right| z_{2}^{(i)} \mid=r_{2}^{(i)}\right\}
$$

with orientation $\left(\arg z_{1}^{(i)}, \arg z_{2}^{(\imath)}\right)$.

If $M_{\imath}$ is the chain

$$
M_{2}=\left\{\left(z_{1}^{(i)}, z_{2}^{(i)}\right)|| z_{1}^{(i)}\left|=r_{1}^{(i)},\right| z_{2}^{(i)} \mid \leqq r_{2}^{(i)}\right\}, \quad r_{1}^{(i)}, r_{2}^{(i)}>0
$$

with orientation ( $\arg z_{1}^{(\imath)}, \Re z_{2}^{(i)}, \mathfrak{J} z_{2}^{(i)}$ ) ( $\Re z$ and $\mathfrak{\Im} z$ denote the real part and the imaginary part of $z$ respectively), then the boundary of $M_{\imath}$ is $N_{\imath}$.

$$
\partial M_{\imath}=N_{\imath}
$$

We have

\section{Lemma 3.2.}

(1) Let $\nu_{\imath}$ be the homology class of $N_{\imath}$ in $H_{2}\left(X_{n} \backslash D\right.$; Z) and $\mu_{\imath}$ the homology class of $M_{\imath}$ in $H_{3}\left(X_{n}, X_{n} \backslash D ; \boldsymbol{Z}\right)$. Then we have

(i) $\nu_{i}=\partial_{*}\left(\mu_{i}\right)$,

(ii) $\nu_{\imath}=-\nu_{i+1}, \mu_{1}=-\mu_{\imath+1}\left(1 \leqq i \leqq 4, \nu_{5}=\nu_{1}, \mu_{5}=\mu_{1}\right)$.

(2) $H_{3}\left(X_{n}, X_{n} \backslash D ; \boldsymbol{Z}\right) \cong \boldsymbol{Z}$, generated by $\mu_{\imath}$.

Proof. By (2.5), (3.6), and (3.7), we have (1). It follows from the duality theorem that

$$
\begin{aligned}
H_{3}\left(X_{n}, X_{n} \backslash D ; \boldsymbol{Z}\right) & \cong H^{1}(D ; \boldsymbol{Z}) \\
& \cong H_{1}(D ; \boldsymbol{Z})^{*} \\
& \cong \boldsymbol{Z} .
\end{aligned}
$$

Lel $\gamma_{\imath}$ be an injective path in $D_{\imath}$ from $D_{\imath-1} \cap D_{\imath}$ to $D_{i} \cap D_{\imath+1}\left(D_{0}=D_{4}\right)$ and $\gamma=$ 
$\gamma_{1}+\cdots+\gamma_{4}$. Then $H_{1}(D ; \boldsymbol{Z})$ is generated by the homology class of $\gamma$. Since the intersection number $M_{i} \cdot \gamma=(-1)^{i}, \mu_{\imath}$ is a generator of $H_{3}\left(X_{n}, X_{n} \backslash D ; \boldsymbol{Z}\right)$ by Poincaré duality.

The Poincaré duality yields a canonical isomorphism

$$
H_{c}^{2}\left(X_{n} \backslash D ; \boldsymbol{Z}\right) \cong H_{2}\left(X_{n} \backslash D ; \boldsymbol{Z}\right),
$$

where $H_{c}^{2}\left(Y_{n} \backslash D ; Z\right)$ is the second cohomology group of $X_{n} \backslash D$ with compact supports. This duality induces the intersection product on $H_{2}\left(X_{n} \backslash D ; \boldsymbol{Z}\right)$

Proposition 3.3. Let $\nu=\partial_{*}(\mu)$ be the image of a generator $\mu$ of $H_{3}\left(X_{n}, Y_{n} \backslash\right.$ $D ; \boldsymbol{Z})$ and $\alpha_{\imath}, 1 \leqq i \leqq n-1$, the class of $\Gamma_{\imath, \imath+1}$ in $H_{2}\left(X_{n} \backslash D ; \boldsymbol{Z}\right)$. Then $H_{2}\left(X_{n} \backslash D ; \boldsymbol{Z}\right)$ is generated by $\nu, \alpha_{1}, \cdots, \alpha_{n-1}$ and the intersection pairing is given by

$$
\begin{aligned}
& \nu^{2}=0, \quad \nu \cdot \alpha_{\imath}=0(1 \leqq i \leqq n-1) \\
& \alpha_{i} \cdot \alpha_{j}= \begin{cases}-2, & \text { if } i=j, \\
1, & \text { if }|i-j|=1, \\
0, & \text { otherwise. }\end{cases}
\end{aligned}
$$

Proof. Since

$$
\Pi=\left\{i_{*}\left(\alpha_{i}\right)=e_{\imath}-e_{\imath+1} \mid 1 \leqq i \leqq n-1\right\}
$$

is a basis of the lattice $Q$ (Proposition 3.1), we have, by (3.3),

$$
H_{2}\left(X_{n} \backslash D ; \boldsymbol{Z}\right) \cong \boldsymbol{Z} \boldsymbol{\nu} \boldsymbol{Z} \boldsymbol{\alpha}_{1} \oplus \cdots \oplus \boldsymbol{Z} \boldsymbol{\alpha}_{n-1} .
$$

The intersection numbers of these generators are given as follows: since $i_{*}(\nu)$ $=0$, we have

$$
\begin{aligned}
\nu \cdot \nu & =i_{*}(\nu) \cdot i_{*}(\nu)=0, \\
\nu \cdot \alpha_{i} & =i_{*}(\nu) \cdot i_{*}\left(\alpha_{i}\right)=0, \\
\alpha_{\imath} \cdot \alpha_{j} & =i_{*}\left(\alpha_{i}\right) \cdot i_{*}\left(\alpha_{\jmath}\right) \\
& =\left(e_{i}-e_{i+1}\right) \cdot\left(e_{j}-e_{j+1}\right) \\
& = \begin{cases}-2, & \text { if } i=j, \\
1, & \text { if }|i-j|=1, \\
0, & \text { otherwise. }\end{cases}
\end{aligned}
$$

\section{§4. Torelli Theorem for the Pairs $\left(X_{n}, D\right)$}

We now prove a Torelli theorem for the pairs $\left(X_{n}, D\right)$, where $X_{n}(n \geqq 2)$ is the blowing up of $\Sigma_{n}$ at $n$ points $P_{1}, \cdots, P_{n}$ in general position and $D \in \mathfrak{D}\left(X_{n}\right)$ 
is a marked anticanonical divisor on $X_{n}$. The formulation is similar to that of [7].

Let $\mu$ be a generator of $H_{3}\left(X_{n}, X_{n} \backslash D ; \boldsymbol{Z}\right)$ and

$$
\nu=\partial_{*}(\mu) \text {. }
$$

There exists a unique meromorphic 2-form $\omega_{\nu}$ on $X_{n}$ such that $\omega_{\nu}$ has poles only along $D$ and $\omega_{\nu}(\nu)=1$. By Lemma 3.2, $\nu$ is the homology class of $N_{1}$ or $N_{2}$ (see (3.5)). If $\nu$ is the homology class of $N_{\imath}$, then

$$
\omega_{\nu}=\frac{1}{(2 \pi \sqrt{-1})^{2}} \frac{d z_{1}^{(i)} \wedge d z_{2}^{(i)}}{z_{1}^{(i)} z_{2}^{(i)}} \quad \text { for } i=1,2
$$

in a neighbourhood of $D_{\imath} \cap D_{\imath+1}$, where $D_{1}=\bar{F}_{1}, D_{2}=\bar{C}$, and $D_{3}=\bar{F}_{2}$. The residue map gives the 1 -form

$$
\operatorname{Res}_{\bar{c}} \omega_{\nu}=\frac{1}{(2 \pi \sqrt{-1})^{2}} \frac{d z_{1}^{(i)}}{z_{1}^{(i)}}
$$

on $\bar{C}$.

Lemma 4.1. Let $\Gamma_{\imath, j}$ be the 2-cycle defined by (3.4). Let $\nu=\partial_{*}(\mu) \equiv$ $H_{2}\left(X_{n} \backslash D ; \boldsymbol{Z}\right)$ is the homology class of $N_{r}(r=1$ or 2$)$, then

$$
\exp \left(2 \pi \sqrt{-1} \int_{\Gamma_{\imath, j}} \omega_{\nu}\right)= \begin{cases}{\left[\bar{F}_{1} \cap \bar{C}, \bar{F}_{2} \cap \bar{C} ; E_{j 1} \cap \bar{C}, E_{\imath} \cap \bar{C}\right],} & \text { if } r=1, \\ {\left[\bar{F}_{2} \cap \bar{C}, \bar{F}_{1} \cap \bar{C} ; E_{j} \cap \bar{C}, E_{\imath} \cap \bar{C}\right],} & \text { if } r=2,\end{cases}
$$

where $\left[Q_{1}, Q_{2} ; Q_{3}, Q_{4}\right]$ denotes the cross ratio of the points $Q_{1}, Q_{2}, Q_{3}$, and $Q_{4}$ on $\boldsymbol{P}^{1}$.

Proof. Since $E_{\imath}$ and $E_{\jmath}$ are the inverse image of the points $P_{\imath}$ and $P_{\jmath}$ respectively, we have

Therefore

$$
\int_{E_{\imath} \backslash\left(E_{\imath} \cap T\right)} \omega_{\nu}=\int_{E_{\jmath} \backslash(E, \cap T)} \omega_{\nu}=0
$$

$$
\int_{\Gamma_{\imath, j}} \omega_{\nu}=\int_{\partial T_{1 \gamma}} \omega_{\nu}
$$

By the residue formula, we have

$$
\begin{aligned}
\int_{\left.\partial T_{\mid \gamma}\right|_{\nu}} \omega_{\nu} & 2 \pi \sqrt{-1} \int_{r} \operatorname{Res}_{\bar{C}} \omega_{\nu} \\
& =\frac{1}{2 \pi \sqrt{-1}} \int_{r} \frac{d z_{1}^{(r)}}{z_{1}^{(r)}} \\
& =\frac{1}{2 \pi \sqrt{-1}} \int_{t_{i}}^{t_{j}} \frac{d z_{1}^{(r)}}{z_{1}^{(r)}} \\
& =\frac{1}{2 \pi \sqrt{-1}} \log \frac{t_{j}}{t_{\iota}}(\bmod \boldsymbol{Z}),
\end{aligned}
$$


where $t_{\imath}$ and $t_{j}$ are the affine coordinates of the points $E_{\imath} \cap \bar{C}$ and $E_{\jmath} \cap \bar{C}$ respectively $\left(\bar{F}_{r} \cap \bar{C}=0\right)$. Then we have

$$
\begin{aligned}
\exp \left(2 \pi \sqrt{-1} \int_{\Gamma_{i, j}} \omega_{\nu}\right) & =\frac{t_{j}}{t_{\imath}} \\
& =\left[(1: 0),(0: 1) ;\left(1: t_{j}\right),\left(1: t_{i}\right)\right] .
\end{aligned}
$$

Thus lemma follows.

We shall define a character $\chi_{\nu}: Q \rightarrow C^{*}$ by

$$
\begin{aligned}
\chi_{\nu}\left(i_{*}[\Gamma]\right)=\exp \left(2 \pi \sqrt{-1} \int_{\Gamma} \omega_{\nu}\right), & \Gamma \in H_{2}\left(X_{n} \backslash D ; \boldsymbol{Z}\right) . \\
H_{2}\left(X_{n} \backslash D ; \boldsymbol{Z}\right) & \stackrel{i_{*}}{\longrightarrow} Q \\
\exp 2 \pi \sqrt{-1} \int_{[\cdot]} \omega_{\nu} \downarrow & \downarrow^{\chi_{\nu}} \\
C^{*} & =C^{*}
\end{aligned}
$$

Notation 4.2. Let $\nu$ be the homology class of $N_{r}(r=1,2)$. We shall introduce the marking of $\bar{F}_{1}, \bar{F}_{2}$ in the following way:

$$
\left\{\begin{array}{lll}
\bar{F}_{0}=\bar{F}_{1}, & \bar{F}_{\infty}=\bar{F}_{2}, & \text { in case } \nu=\left[N_{1}\right], \\
\bar{F}_{0}=\bar{F}_{2}, & \bar{F}_{\infty}=\bar{F}_{1}, & \text { if case } \nu=\left[N_{2}\right] .
\end{array}\right.
$$

Then Lemma 4.1 says that

$$
\exp \left(2 \pi \sqrt{-1} \int_{\Gamma_{\imath, \jmath}} \omega_{\nu}\right)=\left[\bar{F}_{0} \cap \bar{C}, \bar{F}_{\infty} \cap \bar{C} ; E_{\jmath} \cap \bar{C}, E_{\imath} \cap \bar{C}\right]
$$

We now have the Torelli theorem for the pairs $\left(X_{n}, D\right)$.

Theorem 4.3. Let $p: X_{n} \rightarrow \Sigma_{n}$ (resp. $p^{\prime}: X_{n}^{\prime} \rightarrow \Sigma_{n}$ ) be the morphism obtained by blowing up $n$ points $P_{1}, \cdots, P_{n}$ (resp. $\left.P_{1}^{\prime}, \cdots, P_{n}^{\prime}\right)$ in general position and $D E=$ $\grave{D}\left(X_{n}\right)$ (resp. $\left.D^{\prime}=\emptyset\left(X_{n}^{\prime}\right)\right)$ with the marking $D=\bar{F}_{0}+\bar{F}_{\infty}+\bar{S}+\bar{C}$ (resp. $D^{\prime}=\bar{F}_{0}^{\prime}+\bar{F}_{\infty}^{\prime}+$ $\left.\bar{S}^{\prime}+\bar{C}^{\prime}\right)$ associated with the homology class $\nu \in H_{2}\left(X_{n} \backslash D ; Z\right.$ ) (resp. $\nu^{\prime} \in$ $H_{2}\left(X_{n}^{\prime} \backslash D^{\prime} ; Z\right)$ ) (see Notation 4.2). Let $\chi_{\nu}\left(\right.$ resp. $\left.\chi_{\nu^{\prime}}^{\prime}\right)$ be the character of the root lattice defined by (4.3). If $\varphi: H_{2}\left(X_{n} ; \boldsymbol{Z}\right) \rightarrow H_{2}\left(X_{n}^{\prime} ; \boldsymbol{Z}\right)$ is an isometry such that

(1) $\varphi\left(\left[\bar{F}_{0}\right]\right)=\left[\bar{F}_{0}^{\prime}\right], \varphi\left(\left[\bar{F}_{\infty}\right]\right)=\left[\bar{F}_{\infty}^{\prime}\right], \varphi([\bar{C}])=\left[\bar{C}^{\prime}\right], \varphi([\bar{S}])=\left[\bar{S}^{\prime}\right]$,

(2) $\varphi^{*}\left(\chi_{\nu}^{\prime}\right)=\chi_{\nu}$,

then there exists an isomorphism $\Phi: X_{n} \rightarrow X_{n}^{\prime}$ which induces $\varphi$ and maps $\bar{F}_{0}$ to $\bar{F}_{0}^{\prime}, \bar{F}_{\infty}$ to $\bar{F}_{\infty}^{\prime}, \bar{C}$ to $\bar{C}$, and $\bar{S}$ to $\bar{S}^{\prime}$.

Proof. Let $Q$ (resp. $Q^{\prime}$ ) and $R$ (resp. $R^{\prime}$ ) be the root lattice and the root system defined in Section 3. It follows from the condition (1) and the Proposition 3.1 that

$$
\varphi(Q)=Q^{\prime}, \quad \varphi(R)=R^{\prime}
$$


Let $E_{\imath}=p^{-1}\left(P_{\imath}\right)$ and $e_{\imath}$ its class in $H_{2}\left(X_{n} ; Z\right)$. By $(1), e_{\imath}^{\prime}=\varphi\left(e_{\imath}\right)$ is the class of the exceptional curve on $X_{n}^{\prime}$ and let $E_{i}^{\prime}$ be the exceptional curve for $p^{\prime}$ corresponding to the class $e_{2}^{\prime}$. We change the suffixes of $P_{1}^{\prime}, \cdots, P_{n}^{\prime}$ in such a way that $E_{\imath}^{\prime}=\left(p^{\prime}\right)^{-1}\left(P_{2}^{\prime}\right)$. It follows from the condition (2) and (4.4) that

$$
\left.\left[F_{0^{\prime}}\right\urcorner C, F_{\infty} \cap C ; P_{j} \cap C, P_{\imath^{\prime}} \cap C\right]=\left[F_{0}^{\prime} \cap C^{\prime}, F_{\infty}^{\prime} \cap C^{\prime} ; P_{j}^{\prime} \cap C^{\prime}, P_{i}^{\prime} \cap C^{\prime}\right] \text {. }
$$

Thus the theorem follows from the Proposition 2.6.

\section{§5. A Family of the Hirzebruch Surfaces with $n$ Points Blown up}

Let $T$ be a maximal torus of $S L(n, C)(n \geqq 2)$ and $W$ the Weyl group of $S L(n, C)$, which is isomorphic to the symmetric group $\Xi_{n}$ of degree $n$. The quotient space $S=T / W$ is isomorphic to $C^{n-1}$. In this section, we construct a family of Hirzebruch surfaces with $n$ points blown up over $S$. For $t=$ $\left(a_{1}, \cdots, a_{n-1}\right) \equiv S=C^{n-1}$, put

$$
\begin{aligned}
& f_{t}(x)=x^{n}+a_{1} x^{n-1}+\cdots+a_{n-1} x+1 \\
& \left\{\begin{array}{l}
\mathcal{U}_{1}=\left\{\left(x_{1}, y_{1}\right)(\lambda: \mu)\left(a_{1}, \cdots, a_{n-1}\right) \equiv \boldsymbol{C}^{2} \times \boldsymbol{P}^{1} \times S \mid \lambda y_{1}+\mu f_{t}\left(x_{1}\right)=0\right\} \\
\mathcal{U}_{2}=\left\{\left(x_{2}, y_{2}\right)\left(a_{1}, \cdots, a_{n-1}\right) \subseteq \boldsymbol{C}^{2} \times S \mid x_{2}^{n} f_{t}\left(x_{2}^{-1}\right) \neq 0\right\} \\
\mathcal{U}_{3}=\left\{\left(x_{3}, y_{3}\right)\left(a_{1}, \cdots, a_{n-1}\right) \equiv \boldsymbol{C}^{2} \times S\right\} \\
\mathcal{U}_{4}=\left\{\left(x_{4}, y_{4}\right)\left(a_{1}, \cdots, a_{n-1}\right) \subseteq \boldsymbol{C}^{2} \times S\right\} .
\end{array}\right.
\end{aligned}
$$

The manifold.$t i$ is obtained by glueing $\mathcal{V}_{\iota}, 1 \leqq i \leqq 4$, as follows:

$$
\left\{\begin{array}{l}
x_{1} x_{2}=x_{2} x_{4}=1, \quad x_{2}=x_{3}, \\
y_{2}=x_{1}^{-n} y_{1}, \quad y_{1} y_{4}=1, \quad y_{2} y_{3}=1 .
\end{array}\right.
$$

Let $\varphi: \hat{x} \rightarrow S$ be the projection to $S$.

Remark 5.1. The open sets ' $U_{\imath}$ are glued in the same way as the open sets $U_{\imath}$ of Hirzebruch surface $\Sigma_{n}$ (see (2.4), (2.5)).

Proposition 5.2. Let $\dot{t}$ and $S$ be as above, then $\dot{t}$ is nonsingular. Put

$$
\Delta=\left\{t \in S \mid \text { the equation } f_{\iota}(x)=0 \text { has a multiple root }\right\} \text {. }
$$

Then we have

(1) If $t \in S \backslash \Delta$, the fiber $\mathfrak{x}_{t}=\varphi^{-1}(t)$ is a nonsingular surface. In this case, if $t_{1}, \cdots, t_{n}$ be the roots of the equation $f_{t}(x)=0$, then $\dot{x}_{t}$ is isomorphic to the surface obtained by blowing up

$$
\Sigma_{n}=\left\{\left(\zeta_{0}: \zeta_{1}: \zeta_{2}\right)(s: t) \boxminus \boldsymbol{P}^{2} \times \boldsymbol{P}^{1} \mid s^{n} \zeta_{0}=t^{n} \zeta_{1}\right\}
$$

at the points $\left(1: t_{\imath}^{n}: 0\right)\left(t_{\imath}: 1\right), 1 \leqq i \leqq n$, on the $n$-section $C_{0}$ (see (2.3)). 
(2) If $t \in \Delta$, the fiber $\mathfrak{x}_{t}$ has singularities. Put

$$
f_{t}(x)=\left(x-t_{1}\right)^{k_{1}} \cdots\left(x-t_{r}\right)^{k_{r}}, \quad t_{\imath} \neq t_{j}(i \neq j) .
$$

Then $\mathfrak{X}_{t}$ has simple singularities of type $A_{k_{i^{-1}}}, 1 \leqq i \leqq r$.

Proof. For $i \neq 1, \mathcal{U}_{i}$ has no singular point. Let $\mathscr{W}_{1}$ (resp. $\mathscr{W}_{2}$ ) be the open set defined by $\lambda \neq 0$ (resp. $\mu \neq 0$ ) in $\vartheta_{1}$.

Then

$$
\mathcal{V}_{1}=\mathscr{W}_{1} \cup W_{2} .
$$

$$
\left\{\begin{array}{l}
\mathscr{W}_{1} \cong\left\{(x, y, z)\left(a_{1}, \cdots, a_{n-1}\right) \cong C^{3} \times S \mid y+z f_{t}(x)=0\right\} \\
\mathscr{W}_{2} \cong\left\{(x, y, z)\left(a_{1}, \cdots, a_{n-1}\right) \subseteq C^{3} \times S \mid y z+f_{t}(x)=0\right\}
\end{array}\right.
$$

Put

$$
\begin{aligned}
& g_{1}\left(x, y, z, a_{1}, \cdots, a_{n-1}\right)=y+z f_{t}(x), \\
& g_{2}\left(x, y, z, a_{1}, \cdots, a_{n-1}\right)=y z+f_{t}(x),
\end{aligned}
$$

then the rank of the Jacobian matrix of $g_{\imath}$ is not 0 . Thus $\mathfrak{X}$ is nonsingular. (1) Let $t=\left(a_{1}, \cdots, a_{n-1}\right) \equiv S \backslash \Delta$ and $\tilde{U}_{1}=\mathcal{V}_{1} \cap \mathfrak{X}_{t}$, then the first projection $\tilde{U}_{1} \rightarrow V_{1}$ $=C^{2}$ is nothing but the blowing up of $C^{2}$ at the points $P_{2}=\left(t_{i}, 0\right), 1 \leqq i \leqq n$, where $t_{1}, \cdots, t_{n}$ are the roots of $f_{t}(x)=0$. If we identify $V_{1}$ with the open set $U_{1}$ of $\Sigma_{n}$ (see (2.4)), the point $P_{\imath}=\left(t_{i}, 0\right) \subseteq V_{1}$ corresponds to the point $\left(1: t_{i}^{n}: 0\right)$ $\left(t_{2}: 1\right) \in \Sigma_{n}$. Thus we have (1) by Remark 5.1. (2) Let $t \in \Delta$ and put

$$
\begin{aligned}
& \tilde{U}_{1}=\mathcal{U}_{1} \cap \mathfrak{X}_{t}, \\
& W_{1}=\mathscr{W}_{1} \cap \mathfrak{X}_{t}, \\
& W_{2}=\mathscr{W}_{2} \cap \mathfrak{X}_{t} .
\end{aligned}
$$

Then $\tilde{U}_{1}=W_{1} \cup W_{2}$. The open set $W_{1}$ has no singularity. Since

$$
y z+\left(x-t_{1}\right)^{k_{1}} \cdots\left(x-t_{r}\right)^{k} r=0
$$

is the defining equation of $W_{2}$ and $t_{2} \neq t_{j}$ for $i \neq j$, we can take a neighbourhood around $x=t_{i}$ such that

$$
\prod_{\substack{j=1 \\ j=\imath}}^{r}\left(x-t_{j}\right)^{k j} \neq 0 .
$$

Thus we can choose a local coordinate

$$
x^{\prime}=\left(x-t_{i}\right)\left(\prod_{\substack{j=1 \\ j \neq i}}^{r}\left(x-t_{j}\right)^{k_{j}}\right)^{1 / k_{i}} .
$$

Then we have

$$
y z-x^{\prime k} i=0 .
$$

This has the simple singularity of type $A_{k_{i-1}}$ at the origin. 
We next consider a meromorphic 2-form $\omega$ on $\mathfrak{X}$ defined by

$$
\omega=\frac{(-1)^{i-1}}{(2 \pi \sqrt{-1})^{2}} \frac{d x_{i} \wedge d y_{i}}{x_{i} y_{i}} \quad \text { on } \vartheta_{i}, 1 \leqq i \leqq 4 .
$$

Let $\mathfrak{D}$ be the pole divisor of $\omega$ and $\mathfrak{D}_{t}=\mathfrak{D} \cap \mathfrak{X}_{t}$ for $t . \equiv S$.

Proposition 5.3. For $t \in S \backslash \Delta$, we have $\mathfrak{D}_{t}=\bar{F}+\bar{F}^{\prime}+\bar{S}+\bar{C}_{0}$, where $\bar{F}, \bar{F}^{\prime}, \bar{S}$, and $\bar{C}_{0}$ are the strict transforms of the divisors $F, F^{\prime}, S$, and $C_{0}$ on $\Sigma_{n}$ defined in (2.2) and (2.3).

Proof. Let $t=\left(a_{1}, \cdots, a_{n-1}\right) \in S \backslash \Delta$ and $U_{\imath}=\mathcal{V}_{\imath} \cap \mathfrak{X}_{t}$. Let $V_{\imath}(i=1,2)$ be the open subspace of $U_{1}$ defined by

$$
\begin{aligned}
& V_{1}=\left\{\left(x_{1}, y_{1}\right)(\lambda: \mu) E \boldsymbol{C}^{2} \times \boldsymbol{P}^{1} \mid \lambda y_{1}+\mu f_{t}\left(x_{1}\right)=0, \mu \neq 0\right\}, \\
& V_{2}=\left\{\left(x_{1}, y_{1}\right)(\lambda: \mu) \equiv \boldsymbol{C}^{2} \times \boldsymbol{P}^{1} \mid \lambda y_{1}+\mu f_{t}\left(x_{1}\right)=0, \lambda \neq 0\right\},
\end{aligned}
$$

where $f_{t}(x)=x^{n}+a_{1} x^{n-1}+\cdots+a_{n-1} x+1$. Then

$$
U_{1}=V_{1} \cup V_{2} \text {. }
$$

Put $x=x_{1}, y=y_{1}$ and $z=\lambda / \mu$, then

$$
V_{1} \cong\left\{(x, y, z) \subseteq C^{3} \mid y z+f_{t}(x)=0\right\} .
$$

Let $\omega_{t}$ be the restriction of $\omega$ to the fiber $\dot{x}_{t}$. On $V_{1}$,

$$
\omega_{t}=\frac{1}{(2 \pi \sqrt{-1})^{2}} \frac{d x \wedge d y}{x y} .
$$

It follows from $y z+f_{t}(x)=0$ that

$$
\frac{d f_{t}}{d x}(x) d x+z d y+y d z=0 .
$$

Thus we have

$$
\frac{d x \wedge d y}{y}=-\frac{d x \wedge d z}{z}=\frac{d y \wedge d z}{\frac{d f_{t}}{d x}(x)} .
$$

In $V_{1}$, the poles of this form lie on the set

$$
W=\left\{(x, y, z) \in C^{3} \mid y z+f_{t}(x)=0, y=z=\frac{d f_{t}}{d x}(x)=0\right\} .
$$

If $(x, y, z) \in W$, then $f_{t}(x)=0$ by $y=z=0$. Since $t \in S \backslash \Delta, f_{t}(x)=0$ has no multiple root. Thus there is no common root of $f_{t}(x)=0$ and $\left(d f_{t} / d x\right)(x)=0$ and we have $W=\phi$. Hence the 2-form $(d x \wedge d y) / y$ is holomorphic on $V_{1}$ and $\omega_{t}$ has poles only along the divisor defined by $x=0$ on $V_{1}$, which is $\bar{F} \cap V_{1}$.

We next put $z=\mu / \lambda$, then

$$
V_{2} \cong\left\{(x, y, z) \cong C^{3} \mid y+z f_{t}(x)=0\right\}
$$


and

$$
\omega_{t}=\frac{1}{(2 \pi \sqrt{-1})^{2}} \frac{d x \wedge d y}{x y}
$$

on $V_{1}$. We have

$$
z \frac{d f_{t}}{d x}(x) d x+d y+f_{t}(x) d z=0
$$

Thus

$$
\frac{d x \wedge d y}{x y}=\frac{d x \wedge d z}{x z}=-\frac{d y \wedge d z}{x z^{2} \frac{d f}{d x}(x)} .
$$

Since $z=0$ implies $y=0$ and $\bar{F}, \bar{C}_{0}$ are defined by $x=0, z=y=0$ respectively, this form has poles along the divisors $\bar{F} \cap V_{2}$ and $\bar{C}_{0} \cap V_{2}$ on $V_{2}$.

By (5.1)

$$
U_{2} \cong\left\{\left(x_{2}, y_{2}\right) \risingdotseq C^{2} \mid x_{2}^{n} f_{t}\left(x_{2}^{-1}\right) \neq 0\right\}
$$

and

$$
\omega_{t}=-\frac{1}{(2 \pi \sqrt{-1})^{2}} \frac{d x_{2} \wedge d y_{2}}{x_{2} y_{2}}
$$

on $U_{2}$. Since $x_{2}=0$ and $y_{2}=0$ define the divisors $\bar{F}^{\prime}$ and $\bar{C}_{0}$ respectively, $\omega_{t}$ has poles along $\bar{F}^{\prime} \cap U_{2}$ and $\bar{C}_{0} \cap U_{2}$ on $U_{2}$.

Similarly $\omega_{t}$ has poles along $\left(\bar{F}^{\prime} \cup \bar{S}\right) \cap U_{3}$ on $U_{3}$ and $(\bar{F} \cup \bar{S}) \cap U_{4}$ on $U_{4}$.

Remark 5.4. (1) For a semi-universal deformation $\mathfrak{Y}) \rightarrow S$ of simple surface singularity of type $E_{l}(l=6,7,8)$, there is a family $\bar{Y} \longrightarrow S$ whose general fibers are Del Pezzo surfaces and regarded as the compactifications of general fibers of $\mathfrak{Y} \rightarrow S$ ([12]).

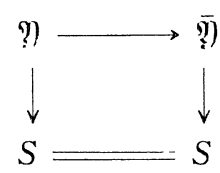

The fiber $\overline{\mathfrak{Y}}_{s}$ is $l$ points blowing up of $\boldsymbol{P}^{2}$ and $C=\overline{\mathfrak{Y}}_{s} \backslash \mathfrak{Y}_{s}$ is an anticanonical divisor of $\overline{\mathfrak{Y}}_{s}$ which is a rational curve with a cusp. It is well known $([5][8])$ that

$$
R=\left\{\alpha \equiv H_{2}\left(\bar{Q}_{s} ; Z\right) \mid \alpha \cdot[C]=0, \alpha \cdot \alpha=-2\right\}
$$

is a root system of type $E_{l}$ in $H_{2}\left(\overline{\mathscr{V}}_{s} ; \boldsymbol{Z}\right)^{\perp} \otimes_{\boldsymbol{Z}} \boldsymbol{R}$, where $[C]$ is the class of the curve $C$ and $H_{2}\left(\overline{\mathfrak{V}}_{s} ; \boldsymbol{Z}\right)^{\perp}$ is the orthogonal complement of $C$ in $H_{2}\left(\overline{\mathfrak{Q}}_{s} ; \boldsymbol{Z}\right)$.

On the other hand, for a semi-universal deformation $3 \rightarrow S$ of simple surface singularity of type $A_{n-1}$, we can construct a similar family $\overline{8} \rightarrow S$ as follows : Put $S=C^{n-1}$ and for $t=\left(a_{1}, \cdots, a_{n-1}\right) \in S$, put

$$
g_{t}(x)=x^{n}+a_{1} x^{n-2}+\cdots+a_{n-1} .
$$


Let

$$
\left\{\begin{array}{l}
\mathscr{V}_{1}=\left\{\left(x_{1}, y_{1}\right)(\lambda: \mu)\left(a_{1}, \cdots, a_{n-1}\right) \subseteq \boldsymbol{C}^{2} \times \boldsymbol{P}^{1} \times S \mid \lambda y_{1}+\mu g_{t}\left(x_{1}\right)=0\right\} \\
\mathscr{V}_{2}=\left\{\left(x_{2}, y_{2}\right)\left(a_{1}, \cdots, a_{n-1}\right) \in \boldsymbol{C}^{2} \times S \mid x_{2}^{n} g_{t}\left(x_{2}^{-1}\right) \neq 0\right\} \\
\mathscr{V}_{3}=\left\{\left(x_{3}, y_{3}\right)\left(a_{1}, \cdots, a_{n-1}\right) \equiv \boldsymbol{C}^{2} \times S\right\} \\
\mathscr{V}_{4}=\left\{\left(x_{4}, y_{4}\right)\left(a_{1}, \cdots, a_{n-1}\right) \subseteq C^{2} \times S\right\} .
\end{array}\right.
$$

Let $\overline{3}$ be the manifold obtained by glueing $\subset V_{i}, 1 \leqq i \leqq 4$, in the same way as $\vartheta_{\imath}$ (see (5.2)). Let

$$
S^{\prime}=\left\{s \in S \mid \text { the fiber } \overline{3}_{s} \text { is nonsingular }\right\} .
$$

The fiber $\overline{3}_{s}, s \in S^{\prime}$, is isomorphic to the $n$ points blowing up of the Hirzebruch surface. Let 3 be the open subspace of $\mathcal{V}_{1}$ defined by $\mu \neq 0$, then $Z \rightarrow S$ can be regarded as a semi-universal deformation of simple surface singularity of type $1_{n-1}$. The fiber $\overline{3}_{s}$ is a compactification of $3_{s}$. If $s \in S^{\prime}$, the complement $\overline{3}_{s} \backslash \Omega_{s}$ has three components $\bar{F}^{\prime}, \bar{S}$ and $\bar{C}_{0}$, which are the divisors in Proposition 5.3. The classes of $\bar{F}^{\prime}, \bar{S}$ and $\bar{C}_{0}$ are $f, s$ and $n f+s-e_{1}-\cdots-e_{n}$ (see Notation 2.4). For $s \cong S^{\prime}$, let

$$
R=\left\{\alpha \equiv H_{2}\left(\bar{\jmath}_{s} ; Z\right) \mid \alpha \cdot f=\alpha \cdot s=\alpha \cdot\left[\bar{C}_{0}\right]=0, \alpha \cdot \alpha=-2\right\} .
$$

then $R$ is a root system of type $A_{n-1}$ in $H_{2}\left(\bar{B}_{s} ; \boldsymbol{Z}\right)^{\perp} \otimes_{\boldsymbol{Z}} \boldsymbol{R}$ by Proposition 3.1, where $H_{2}\left(\bar{B}_{s}, Z\right)^{\perp}$ is the orthogonal complement of $f, s$, and $\left[\bar{C}_{0}\right]$ in $H_{2}\left(\bar{\Omega}_{s}, Z\right)$ with respect to the intersection pairing.

(2) For $s=\left(a_{1}, \cdots, a_{n-1}\right) \equiv S^{\prime}$ with $a_{n-1} \neq 0$, let

$$
\omega_{s}^{(1)}=\frac{1}{(2 \pi \sqrt{-1})^{2}} \frac{d x_{1} \wedge d y_{1}}{x_{1} y_{1}}
$$

be a 2 -form on $\overline{3}_{s} / \backslash \mathcal{V}_{1}$, which has poles along $\bar{F}^{\prime} \cup \bar{S}$. Extending $\omega_{s}^{(1)}$ to $\overline{3}_{s}$ by the transition functions (5.2), we obtain the 2 -form $\omega_{s}$ on $\overline{3}_{s}$. This is the same 2 -form as in the proof of Proposition 5.3.

§ 6. Monodromy Representation of $\pi_{1}(S \backslash \Delta)$ on $H_{2}\left(\ddot{\mathfrak{X}}_{t} \backslash \mathfrak{D}_{t} ; Z\right)$

Let $\varphi: \mathfrak{X} \rightarrow S$, $\mathfrak{D}$ and $\Delta$ be as in Section 5 . Let

$$
\begin{aligned}
& S^{\prime}=S \backslash \Delta \\
& \mathfrak{X}^{\prime}=\mathfrak{X} \backslash\left(\mathfrak{D} \cup \varphi^{-1}(\Delta)\right) .
\end{aligned}
$$

Then $\varphi: \mathfrak{X}^{\prime} \rightarrow S^{\prime}$ is a locally trivial fiber bundle whose fibers are open surfaces $\mathfrak{X}_{t} \backslash \mathfrak{D}_{t}, t \in S^{\prime}$. The fundamental group $\pi_{1}\left(S^{\prime}, t\right)$ acts on $H_{2}\left(\mathfrak{X}_{t} \backslash \mathfrak{D}_{t} ; \boldsymbol{Z}\right)$ as the monodromy of $\varphi: \mathfrak{X}^{\prime} \rightarrow S^{\prime}$.

Let

$$
T=\left\{\left(t_{1}, \cdots, t_{n}\right) \Subset\left(C^{*}\right)^{n} \mid t_{1} \cdots t_{n}=1\right\}
$$




$$
\tilde{\Delta}=\left\{\left(t_{1}, \cdots, t_{n}\right) \in T \mid \prod_{1 \leqq i<j \leqq n}\left(t_{\imath}-t_{j}\right)=0\right\} .
$$

The symmetric group $\Im_{n}$ of degree $n$ acts on $T$ by permutations of coordinates and its quotient space is isomorphic to $S$ :

$$
S \cong T / \Im_{n} .
$$

By the definition of $\Delta$, we have

$$
S^{\prime} \cong(T \backslash \tilde{\Delta}) / \widetilde{S}_{n}
$$

Let $t$ be the Lie algebra of $T$ and $t_{C}$ its complexification

$$
\begin{aligned}
& \mathrm{t}=\left\{\left(x_{1}, \cdots, x_{n}\right) \equiv \boldsymbol{R}^{n} \mid \sum_{i=1}^{n} x_{i}=0\right\} \\
& \mathrm{t}_{C}=\mathrm{t} \otimes_{R} C .
\end{aligned}
$$

Let

$$
Q=\left\{\left(x_{1}, \cdots, x_{n}\right) \Subset t \mid x_{i} \in Z\right\} .
$$

Then the kernel of exponential mapping exp: $t_{C} \rightarrow T$ is $2 \pi \sqrt{-1} Q$, where

$$
\begin{gathered}
\exp \left(\left(x_{1}, \cdots, x_{n}\right)\right)=\left(e^{x_{1}}, \cdots, e^{x_{n}}\right) . \\
0 \longrightarrow 2 \pi \sqrt{-1} Q \longrightarrow \mathrm{t}_{C} \stackrel{\exp }{\longrightarrow} T \longrightarrow 1 .
\end{gathered}
$$

Therefore we have

$$
S \cong T / \Im_{n} \cong \mathrm{t}_{C} /\left(Q \rtimes \Im_{n}\right),
$$

where $\mathfrak{S}_{n}$ acts on $\mathrm{t}_{C}$ by permutations of coordinates and $Q$ acts on $\mathrm{t}_{C}$ by translations :

$$
\alpha \cdot x=x+2 \pi \sqrt{-1} \alpha, \quad\left(\alpha \subseteq Q, x \sqsubseteq \mathrm{t}_{C}\right) .
$$

We denote by $\widetilde{W}$ the group $Q \rtimes \Im_{n}$. It is isomorphic to the affine Weyl group of the root system of type $A_{n-1}$ (see [2]). Let

$$
\begin{aligned}
& H_{i, j}=\left\{\left(x_{1}, \cdots, x_{n}\right) \in \mathrm{t} \mid x_{i}=x_{j}\right\}, \\
& L_{i, j}^{k}=\left\{\left(x_{1}, \cdots, x_{n}\right) \in \mathrm{t} \mid x_{i}-x_{j}=k\right\} .
\end{aligned}
$$

where $1 \leqq i<j \leqq n, k \cong Z$. A fundamental region of $\widetilde{W}$ in $t_{C}$ is given by

$$
\mathrm{t}+2 \pi \sqrt{-1} \bar{A}
$$

where

$$
\bar{A}=\left\{\left(x_{1}, \cdots, x_{n}\right) \subseteq \mathrm{t} \mid x_{i} \geqq x_{j} \text { for } i<j, x_{1}-x_{n} \leqq 1\right\} .
$$

Thus each element of $\mathrm{t}_{C}$ is equivalent under $\widetilde{W}$ to just one element of $\mathfrak{t}+2 \pi \sqrt{-1} \bar{A}$.

Let 


$$
\mathrm{t}_{C}{ }^{\prime}=\mathrm{t}_{C} \backslash \underset{\substack{i \leq j, k \\ 1 \leq i<j \leq n \\ k \in Z}}{\bigcup_{i, j}}\left(H_{i, j}+2 \pi \sqrt{-1} L_{i, j}^{k}\right) .
$$

It follows from (6.1) and (6.2) that

$$
S^{\prime}=\mathrm{t}_{c^{\prime}} / \widetilde{W} .
$$

A fundamental region of $\widetilde{W}$ in $t_{C}{ }^{\prime}$ is given by

$$
\mathrm{t}+2 \pi \sqrt{-1} A,
$$

where $A$ is the interior of $\bar{A}$. By (6.3), the hyperplanes $L_{1, n}^{1}, H_{1,2}, \cdots, H_{n-1, n}$ are the walls of $A$. Let us denote $L_{1, n}^{1}$ by $H_{0}$ and $H_{i, i+1}$ by $H_{i}$. Let $w_{0}, w_{1}$, $\cdots, w_{n-1}$ be the orthogonal reflections fixing these hyperplanes with respect to the bilinear form on $t$ given by

$$
\left\langle\left(x_{i}\right),\left(y_{\imath}\right)\right\rangle=\sum_{i=1}^{n} x_{\imath} y_{\imath} .
$$

Let $m_{i j}$ be the order of $w_{\imath} w_{j}$. Then

$$
\begin{cases}m_{i j}=1, & \text { if } i=j, \\ m_{i j}=3, & \text { if }|i-j|=1, \\ m_{i j}=3, & \text { if }(i, j)=(0, n) \text { or }(n, 0), \\ m_{i j}=2, & \text { otherwise. }\end{cases}
$$

The following result has been proved by Nguyễn Viêt Dũng.

Theorem 6.1 ([11]). The fundamental group $\pi_{1}\left(S^{\prime}\right)$ has a presentation with generators $\sigma_{0}, \sigma_{1}, \cdots, \sigma_{n-1}$ and relations:

$$
\underbrace{\sigma_{i} \sigma_{j} \sigma_{\imath} \cdots}_{m_{i j} \text { times }}=\underbrace{\sigma_{j} \sigma_{i} \sigma_{j} \cdots}_{m_{i j} \text { times }}
$$

The loop corresponding to the generator $\sigma_{\imath}$ can be given as follows (see [11]). Let

$$
\omega_{i}=\frac{1}{n}\left(n-i, n-i, \cdots, \stackrel{2}{n-i}_{-}-i, \cdots,-i\right) \in \mathrm{t} .
$$

Then $\omega_{1}, \cdots, \omega_{n-1}$ and $O$ are the vertices of the convex polyhedron $\bar{A}$. Let us denote by $\widetilde{\omega}$ the bary-center of $\bar{A}$ :

$$
\begin{aligned}
\tilde{\omega} & =\frac{1}{n}\left(\omega_{1}+\cdots+\omega_{n-1}\right) \\
& =\frac{1}{2 n}(n-1, n-3, \cdots,-(n-3),-(n-1)) .
\end{aligned}
$$

Let 


$$
\begin{aligned}
a_{i}^{\prime} & =(0, \cdots, 0, \stackrel{\imath}{1},-1,0, \cdots, 0), \quad 1 \leqq i \leqq n-1 \\
\alpha_{0}^{\prime} & =(-1,0, \cdots, 0,1), \\
\widetilde{\omega}^{(i)} & =\frac{1}{n-1} \sum_{\substack{j=1 \\
j=i}}^{n-1} \omega_{j}, \quad 0 \leqq i \leqq n-1 .
\end{aligned}
$$

Then $\widetilde{\omega}^{(\imath)}$ is the bary-center of the face $\bar{A} \cap H_{\imath}, 0 \leqq i \leqq n-1$. Let

$$
\gamma_{i}^{(1)}:[0,1] \longrightarrow \mathrm{t}_{C}
$$

be the path from $2 \pi \sqrt{-1} \tilde{\omega}$ to $\alpha_{i}^{\prime}+2 \pi \sqrt{-1} \tilde{\omega}^{(i)}$ defined by

$$
\gamma_{i}^{(1)}=(1-s)(2 \pi \sqrt{-1} \tilde{\omega})+s\left(\alpha_{i}^{\prime}+2 \pi \sqrt{-1} \widetilde{\omega}^{(i)}\right) .
$$

Let

$$
\gamma_{i}^{(2)}:[0,1] \longrightarrow \mathrm{t}_{C}
$$

be the path from $\alpha_{\imath}^{\prime}+2 \pi \sqrt{-1} \tilde{\omega}^{(\imath)}$ to $w_{\imath}(2 \pi \sqrt{-1} \tilde{\omega})$ defined by

$$
\gamma_{i}^{(2)}=(1-s)\left(\alpha_{i}^{\prime}+2 \pi \sqrt{-1} \tilde{\omega}^{(i)}\right)+s\left(w_{\imath}(2 \pi \sqrt{-1} \tilde{\omega})\right) .
$$

Let us denote by $\gamma_{2}$ the product of $\gamma_{i}^{(1)}$ and $\gamma_{i}^{(2)}$ beginning at $2 \pi \sqrt{-1} \tilde{\boldsymbol{\sigma}}$ and encling at $w_{2}(2 \pi \sqrt{-1} \tilde{\boldsymbol{\omega}})$.

$$
\gamma_{2}=\gamma_{i}^{(1)} \gamma_{i}^{(2)}
$$

The path $\gamma_{2}$ is in $t_{c}{ }^{\prime}$. The image $\bar{\gamma}_{2}$ of $\gamma_{2}$ under the projection $t_{c}{ }^{\prime} \rightarrow S^{\prime}=$ $\mathrm{t}_{c}{ }^{\prime} / \widehat{W}$ gives the generator $\sigma_{\imath}$ of $\pi_{1}\left(S^{\prime}\right)$. When $n=3$, the path $\gamma_{\imath}$ is given as in the following figure: $\mathrm{t}_{C}=\mathrm{t}+2 \pi \sqrt{-1 \mathrm{t}}$
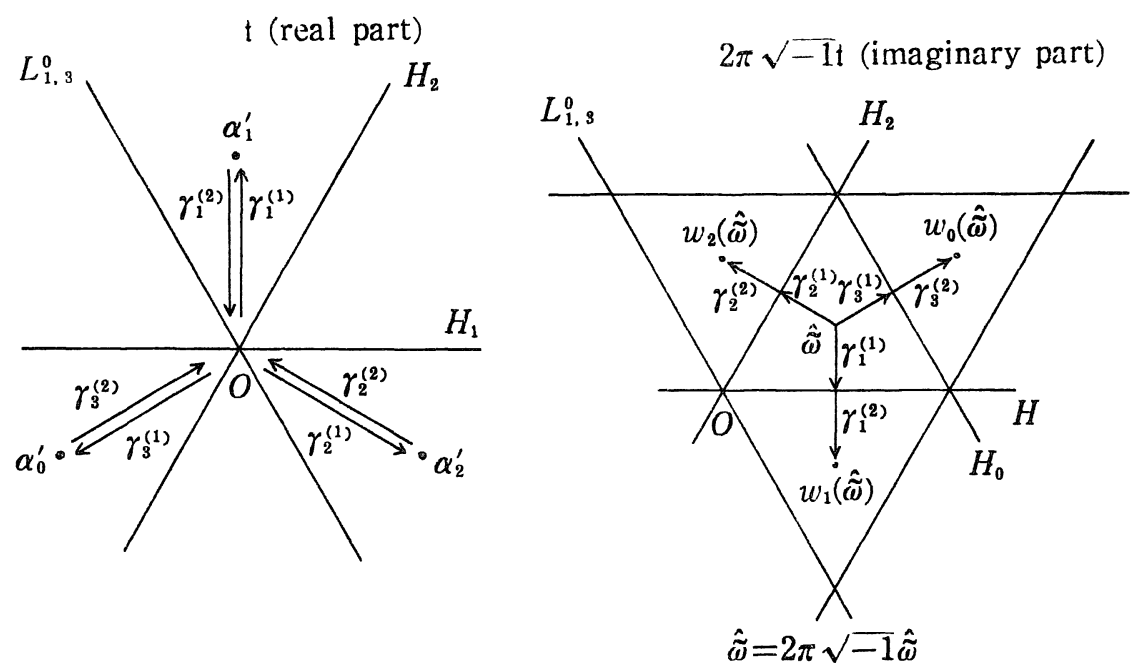

Let $\zeta=e^{2 \pi \sqrt{-1} / 2 n}$ and 


$$
\hat{t}_{0}=\left(\zeta^{n-1}, \zeta^{n-3}, \cdots, \zeta^{-(n-1)}\right) \in T \backslash \tilde{\Delta} .
$$

Then $\hat{t}_{0}=\exp (2 \pi \sqrt{-1} \widetilde{\omega})$. Let $t_{0}$ be the image of $\hat{t}_{0}$ under the quotient mapping $T \rightarrow T / \mathfrak{S}_{n}$. Before describing the monodromy, we shall give 2-cycles of $\mathfrak{X}_{t_{0}}$ whose homology classes are generators of $H_{2}\left(\mathfrak{X}_{t_{0}} \backslash \mathfrak{D}_{t_{0}} ; \boldsymbol{Z}\right)$. It follows from Proposition 5.2 that the fiber $\ddot{\mathfrak{x}}_{t_{0}}$ is the surface obtained by blowing up $\Sigma_{n}$ at the points $\left(1: \zeta^{n(n-22+1)}: 0\right)\left(\zeta^{n-2 i+1}: 1\right), 1 \leqq i \leqq n$. By the arguement in the proof of Proposition 5.2,

$$
U_{1}=U_{1} \cap \mathfrak{X}_{t_{0}}
$$

is the blowing up of $C^{2}$ at the points $P_{\imath}=\left(\zeta^{n-2 i+1}, 0\right), 1 \leqq i \leqq n$,

$$
U_{1} \cong\left\{\left(x_{1}, y_{1}\right)(\lambda: \mu) \subseteq \boldsymbol{C}^{2} \times \boldsymbol{P}^{1} \mid \lambda y_{1}+\mu \prod_{i=1}^{n}\left(x_{1}-\zeta^{n-2 i+1}\right)=0\right\} .
$$

For $\varepsilon>0$, let $N$ be the torus in $U_{1}$ defined by $\left|x_{1}\right|=\left|y_{1}\right|=\varepsilon$ with orientation $\left(\arg x_{1}, \arg y_{1}\right)$. Let $E_{2}$ be the exceptional curve defined by $x_{1}=\zeta^{n-2 i+1}$, $y_{1}=0$ in $U_{1}$. Let $Q V$ be the closed tubular neighborhood of the $x_{1}$-axis in $U_{1}$ such that $\mathcal{V} \cap E_{\iota}$ is a fiber. Let $\tau_{\imath}$ be the path from $\left(\zeta^{n-2 i+1}, 0\right)(1: 0)$ to $\left(\zeta^{n-2(i+1)+1}, 0\right)(1: 0)$ in $U_{1}$ defined by

$$
\tau_{\imath}(s)=\left(e^{-(2 \pi \cdot-\overline{-1} / n) s} \zeta^{n-2 i+1}, 0\right)(1: 0), \quad s \equiv[0,1] .
$$

Then we can construct a 2-cycle $\Gamma_{i, i+1}$ as in Section 3 (see (3.4)):

$$
\Gamma_{i, i+1}=\left.\left(E_{\imath} \backslash\left(E_{\imath} \cap C \mathcal{V}\right)\right) \cup \partial \mathcal{}\right|_{\tau_{i}} \cup\left(E_{i+1} \backslash\left(E_{i+1} \cap C \mathcal{C}\right)\right),
$$

with orientation $\left(\arg x_{1}, \arg y_{1}\right)$.

Let $\nu$ and $\alpha_{\imath}$ are the homology classes of $N$ and $\Gamma_{i, i+1}$ respectively. It follows from Proposition 3.3 that $H_{2}\left(\dot{\mathfrak{x}}_{t_{0}} \backslash \mathfrak{D}_{t_{0}} ; \boldsymbol{Z}\right)$ is generated by $\nu, \alpha_{1}, \cdots, \alpha_{n-1}$.

Theorem 6.2. Let $t_{0} \Xi S^{\prime}$ be the point corresponding to $\tilde{\boldsymbol{\omega}}$. Let

$$
\rho: \pi_{1}\left(S^{\prime}, t_{0}\right) \longrightarrow \operatorname{Aut}\left(H_{2}\left(\mathfrak{X}_{t_{0}} \backslash \mathfrak{D}_{t_{0}} ; Z\right)\right)
$$

be the monodromy of the fibration $\varphi: \mathfrak{X}^{\prime} \rightarrow S^{\prime}$. Let $\nu, \alpha_{1}, \cdots, \alpha_{n-1}$ be the generators of $\left.H_{2}\left(\mathfrak{x}_{t_{0}} \backslash \mathfrak{D}_{t_{0}} ; \boldsymbol{Z}\right)\right)$ and $\sigma_{0}, \sigma_{1}, \cdots, \sigma_{n-1}$ the generators of $\pi_{1}\left(S^{\prime}, t_{0}\right)$ as above. Let $\alpha_{0}=-\nu-\alpha_{1}-\cdots-\alpha_{n-1}$, then $\sigma_{i}$ acts on $H_{2}\left(\mathfrak{X}_{t_{0}} \backslash \mathfrak{D}_{t_{0}} ; \boldsymbol{Z}\right)$ by

$$
\begin{gathered}
\rho\left(\sigma_{i}\right)(x)=x-\frac{2 x \cdot \alpha_{\imath}}{\alpha_{\imath} \cdot \alpha_{\imath}} \alpha_{\imath}, \\
\rho\left(\sigma_{\imath} \sigma_{j}\right)(x)=\rho\left(\sigma_{j}\right) \rho\left(\sigma_{i}\right)(x),
\end{gathered}
$$

where the dot · denote the intersection pairing given in Proposition 3.3. The monodromy group, the image $\rho\left(\pi_{1}\left(S^{\prime}, t_{0}\right)\right)$, is isomorphic to the afjine Weyl group of the root system of type $A_{n-1}$.

Proof. In order to describe the action of $\sigma_{i}$, we consider the parallel displacement of the cycles $N, \Gamma_{1,2}, \cdots, \Gamma_{n-1, n}$ along the path $\bar{\gamma}_{2}$ in $S^{\prime}=(T \backslash \tilde{\Delta}) / \Im_{n}$. 
First we consider the action of $\sigma_{i}$ for $i=1, \cdots, n-1$. The path $\gamma_{i}, 1 \leqq i \leqq n-1$, gives the path in $T \backslash \tilde{\Delta}$ from $\left(\zeta^{n-1}, \zeta^{n-3}, \cdots, \zeta^{-(n-1)}\right)$ to

$$
\left(\zeta^{n-1}, \zeta^{n-3}, \cdots, \zeta^{n-2(i-1)+1}, \zeta^{n-2(i+1)+1}, \zeta^{n-2 i+1}, \zeta^{n-2(i+2)+1}, \cdots, \zeta^{-(n-1)}\right) .
$$

By the construction of the cycles $N$ and $\Gamma_{i, \imath+1}$, the resulting cycles $N^{\prime}$ and $\Gamma_{i, i+1}^{\prime}$ of parallel displacement of $N$ and $\Gamma_{i, i+1}$ along the path $\bar{\gamma}_{i}$ are given by (up to homology):

$$
\left\{\begin{array}{l}
{\left[N^{\prime}\right]=[N]=\nu,} \\
{\left[\Gamma_{i-1, i}^{\prime}\right]=\left[\Gamma_{i-1, i}\right]+\left[\Gamma_{i, i+1}\right]=\alpha_{i-1}+\alpha_{i},} \\
{\left[\Gamma_{i, i+1}^{\prime}\right]=-\left[\Gamma_{i, i+1}\right]=-\alpha_{i},} \\
{\left[\Gamma_{i+1, i+2}^{\prime}\right]=\left[\Gamma_{i, i+1}\right]+\left[\Gamma_{i+1, i+2}\right]=\alpha_{i}+\alpha_{i+1},} \\
{\left[\Gamma_{j, j+1}^{\prime}\right]=\left[\Gamma_{j, j+1}\right]=\alpha_{j} \quad \text { if } j \neq i-1, i, i+1 .}
\end{array}\right.
$$

Thus we have

$$
\rho\left(\sigma_{i}\right)(x)=x-\frac{2 x \cdot \alpha_{i}}{\alpha_{i} \cdot \alpha_{i}} \alpha_{i}, \quad 1 \leqq i \leqq n-1
$$

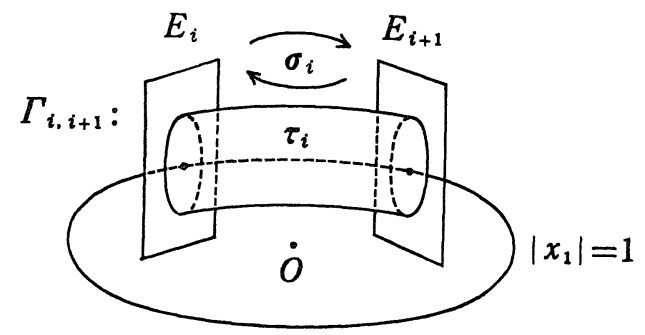

We next describe the action of $\sigma_{0}$. The path $\gamma_{0}$ gives the path in $T \backslash \tilde{\Delta}$ from $\left(\zeta^{n-1}, \zeta^{n-3}, \cdots, \zeta^{-(n-1)}\right)$ to $\left(\zeta^{-(n-1)}, \zeta^{n-3}, \cdots, \zeta^{n-1}\right)$. On the homology we have

$$
\begin{gathered}
{\left[N^{\prime}\right]=[N]=\nu,} \\
{\left[\Gamma_{i, i+1}^{\prime}\right]=\left[\Gamma_{i, i+1}\right]=\alpha_{\imath} \quad \text { if } i=2,3, \cdots, n-2 .}
\end{gathered}
$$

The classes $\left[\Gamma_{1,2}^{\prime}\right]$ and $\left[\Gamma_{n-1, n}^{\prime}\right]$ can be calculated as follows. Let $\tau_{1}^{\prime}$ be the path from $\left(\zeta^{-(n-1)}, 0\right)(1: 0)$ to $\left(\zeta^{n-1}, 0\right)(1: 0)$ in $U_{1}$ defined by

$$
\tau_{1}^{\prime}(s)=\left(e^{-(2 \pi \sqrt{-1} / n) s} \zeta^{-(n-1)}, 0\right)(1: 0), \quad s \leqq[0,1] .
$$

Then the cycle $\Gamma_{1,2}^{\prime}$ is homologous to the cycle

$$
\Gamma^{\prime}=\left.\left(E_{n} \backslash\left(E_{n} \cap \mathcal{V}\right)\right) \cup \partial \mathcal{V}\right|_{\tau_{1}^{\prime} \tau_{1}} \cup\left(E_{2} \backslash\left(E_{2} \cap \mathcal{V}\right)\right) .
$$

The cycle $\Gamma^{\prime}+\Gamma_{2,3}+\cdots+\Gamma_{n-1, n}$ is homologous to $-N$. Hence we have 


$$
\begin{aligned}
\Gamma_{1,2}^{\prime} & =-[N]-\left[\Gamma_{2,3}\right]-\cdots-\left[\Gamma_{n-1, n}\right] \\
& =-\left(\nu+\alpha_{2}+\cdots+\alpha_{n-1}\right) .
\end{aligned}
$$

Similarly we have

$$
\begin{aligned}
\Gamma_{n-1, n}^{\prime} & =-[N]-\left[\Gamma_{1,2}\right]-\cdots-\left[\Gamma_{n-2, n-1}\right] \\
& =-\left(\nu+\alpha_{1}+\cdots+\alpha_{n-2}\right) .
\end{aligned}
$$

Thus we have

$$
\rho\left(\sigma_{0}\right)(x)=x-\frac{2 x \cdot \alpha_{0}}{\alpha_{0} \cdot \alpha_{0}} \alpha_{0},
$$

where $\alpha_{0}=-\left(\nu+\alpha_{1}+\cdots+\alpha_{n-1}\right)$.

The elements $\rho\left(\sigma_{i}\right), 0 \leqq i \leqq n-1$, have the same relations as $w_{i}, 0 \leqq i \leqq n-1$. Thus $\rho\left(\pi_{1}\left(S^{\prime}, t_{0}\right)\right)$ is isomorphic to the affine Weyl group of the root system of type $A_{n-1}$.

The fundamental group $\pi_{1}\left(S^{\prime}, t_{0}\right)$ acts on $\operatorname{Hom}_{\boldsymbol{Z}}\left(H_{2}\left(\mathfrak{X}_{t_{0}} \backslash \mathfrak{D}_{t_{0}} ; \boldsymbol{Z}\right), \boldsymbol{C}\right)$ by

$$
(\sigma \cdot f)(x)=f\left(\rho(\sigma)^{-1}(x)\right) \quad \sigma \in \pi_{1}\left(S^{\prime}, t_{0}\right) .
$$

Let

$$
\Omega=\left\{f \in \operatorname{Hom}_{\boldsymbol{Z}}\left(H_{2}\left(\mathfrak{X}_{t_{0}} \backslash \mathscr{D}_{t_{0}} ; \boldsymbol{Z}\right), \boldsymbol{C}\right) \mid f(\nu)=1\right\} .
$$

Since $\nu$ is fixed by the action of $\pi_{1}\left(S^{\prime}, t_{0}\right), \Omega$ is stable under the action of $\pi_{1}\left(S^{\prime}, t_{0}\right)$. Let

$$
\rho^{*}: \pi_{1}\left(S^{\prime}, t_{0}\right) \longrightarrow \operatorname{Aut}(\Omega)
$$

be the homomorphism defined by this action.

Let $\nu^{*}$ and $\alpha_{\imath}^{*}, 1 \leqq i \leqq n-1$, be the elements of $\operatorname{Hom}_{\boldsymbol{Z}}\left(H_{2}\left(\mathfrak{X}_{t_{0}} \backslash \mathfrak{D}_{t_{0}} ; \boldsymbol{Z}\right), \boldsymbol{C}\right)$ defined by

$$
\begin{aligned}
& \nu^{*}(x)= \begin{cases}1, & \text { if } x=\nu, \\
0, & \text { otherwise, }\end{cases} \\
& \alpha_{\imath}^{*}(x)=\alpha_{\imath} \cdot x .
\end{aligned}
$$

Let $f \in \Omega$. Since $f(\nu)=1, f$ has the form $f=\nu^{*}+\sum_{\imath=1}^{n-1} a_{\imath} \alpha_{\imath}^{*}\left(a_{\imath} \equiv \boldsymbol{C}\right)$. Let $V^{*}$ be the vector space $\sum_{i=1}^{n-1} \boldsymbol{R} \alpha_{i}^{*}$, then

$$
\Omega=\left\{\nu^{*}+v_{1}^{*}+\sqrt{-1} v_{2}^{*} \mid v_{\imath}^{*} \subseteq V^{*}\right\} .
$$

We shall define a non-degenerate bilinear form on $V^{*}$ by

$$
\left\langle x^{*}, y^{*}\right\rangle=\left(\sum_{i=1}^{n-1} x_{i} \alpha_{i}\right) \cdot\left(\sum_{i=1}^{n-1} y_{i} \alpha_{i}\right),
$$

where $x^{*}=\left(\sum_{i=1}^{n-1} x_{\imath} \alpha_{\imath}^{*}\right)$ and $y^{*}=\left(\sum_{\imath=1}^{n-1} y_{\imath} \alpha_{\imath}^{*}\right)$. Let us write $f=\nu^{*}+v_{f, R}^{*}+$ $\sqrt{-1} v_{f, I}^{*} \in \Omega\left(v_{f, R}^{*}, v_{f, I}^{*} \boxminus V^{*}\right)$. 
Let $w_{\alpha_{i}^{*}}, 1 \leqq i \leqq n-1$, be the reflection in the hyperplane orthogonal to $\alpha_{i}^{*}$ and $W^{*}$ the group generated by $w_{\alpha_{1}^{*}}, \cdots, w_{\alpha_{n-1}^{*}}$. Let $R^{*}=W^{*}\left(\left\{\alpha_{1}^{*}, \cdots, \alpha_{n-1}^{*}\right\}\right)$. Then $R^{*}$ forms a root system of type $A_{n-1}$ in $V^{*}$. For $\alpha^{*} \subseteq R^{*}$ and $k \in Z$, let

$$
L_{\alpha^{*}, k}^{*}=\left\{v^{*} \in V^{*} \mid\left\langle\alpha^{*}, v^{*}\right\rangle=k\right\} .
$$

The group $\widetilde{W}^{*}$ generated by the reflections $w_{\alpha^{*}, k}$ in the hyperplanes $L_{\alpha^{*}, k}^{*}\left(\alpha^{*}\right.$ $\left.\Leftarrow R^{*}, k \in \boldsymbol{Z}\right)$ is isomorphic to the affine Weyl group of the root system of type $A_{n-1}$.

Corollary 6.3. The fundamental group $\pi_{1}\left(S^{\prime}, t_{0}\right)$ acts on $\Omega$ as affine transformations :

$$
\rho^{*}\left(\sigma_{i}\right)(f)= \begin{cases}\nu^{*}+w_{\alpha_{i}^{*}}\left(v_{f, R}^{*}\right)+\sqrt{-1} w_{\alpha_{i}^{*}}\left(v_{f, I}^{*}\right), & \text { if } 1 \leqq i \leqq n-1, \\ \nu^{*}+w_{\beta^{*, 1}}\left(v_{f, R}^{*}\right)+\sqrt{-1} w_{\beta^{*, 0}}\left(v_{f, I}^{*}\right), & \text { if } i=0,\end{cases}
$$

where $f=\nu^{*}+v_{f, R}^{*}+\sqrt{-1} v_{f, I}^{*} \equiv \Omega\left(v_{f, R}^{*}, v_{f, I}^{*} \subseteq V^{*}\right), \beta^{*}=-\left(\alpha_{1}^{*}+\cdots+\alpha_{n-1}^{*}\right)$. The image of $\rho^{*}$ is isomorphic to the affine Weyl group of the root system of type $A_{n-1}$.

Proof. For $i \geqq 1$ and $f \in \Omega$,

$$
\begin{aligned}
\left(\rho^{*}\left(\sigma_{i}\right) f\right)(x) & =f\left(\rho^{*}\left(\sigma_{i}\right)^{-1}(x)\right) \\
& =f\left(x+\left(\alpha_{i} \cdot x\right) \alpha_{\imath}\right) \quad(\text { by Theorem 6.2) } \\
& =f(x)+f\left(\alpha_{i}\right)\left(\alpha_{i} \cdot x\right) \\
& =f(x)+\left(\left\langle v_{f, R}^{*}, \alpha_{i}^{*}\right\rangle+\sqrt{-1}\left\langle v_{f, I}^{*}, \alpha_{i}^{*}\right\rangle\right) \alpha_{i}^{*}(x) .
\end{aligned}
$$

Thus we have

$$
\rho^{*}\left(\sigma_{i}\right) f=2^{*}+\left(v_{f, R}^{*}+\left\langle v_{f, R}^{*}, \alpha_{i}^{*}\right\rangle \alpha_{i}^{*}\right)+\sqrt{-1}\left(v_{f, I}^{*}+\left\langle v_{f, I}^{*}, \alpha_{i}^{*}\right\rangle \alpha_{i}^{*}\right) .
$$

This implies that $\sigma_{i}$ acts on $V^{*}$ and $\sqrt{-1} V^{*}$ as the reflection in the hyperplane $L_{i}^{*}$ orthogonal to $\alpha_{i}^{*}(1 \leqq i \leqq n-1)$. For $i=0$, let $\beta=-\left(\alpha_{1}+\cdots+\alpha_{n-1}\right)$ and $\beta^{*}=-\left(\alpha_{1}^{*}+\cdots+\alpha_{n-1}^{*}\right)$. Since

$$
\begin{aligned}
f\left(\alpha_{0}\right) & =\left(\nu^{*}+v_{f, R}^{*}+\sqrt{-1} v_{f, I}^{*}\right)(-\nu+\beta) \\
& =-1+\left\langle v_{f, R}^{*}, \beta^{*}\right\rangle+\sqrt{-1}\left\langle v_{f, I}^{*}, \beta^{*}\right\rangle
\end{aligned}
$$

and

$$
\alpha_{0} \cdot x=\beta \cdot x=\beta^{*}(x),
$$

we have

$$
\begin{aligned}
\left(\rho^{*}\left(\sigma_{0}\right) f\right)(x) & =f(x)+f\left(\alpha_{0}\right)\left(\alpha_{0} \cdot x\right) \\
& =f(x)+\left(-1+\left\langle v_{f, R}^{*}, \beta^{*}\right\rangle+\sqrt{-1}\left\langle v_{f, I}^{*}, \beta^{*}\right\rangle\right) \beta^{*}(x) .
\end{aligned}
$$

Thus we have 


$$
\rho^{*}\left(\sigma_{0}\right) f=\nu^{*}+\left(v_{f, R}^{*}+\left(\left\langle v_{f, R}^{*}, \beta^{*}\right\rangle-1\right) \beta^{*}\right)+\sqrt{-1}\left(v_{f, I}^{*}+\left\langle v_{f, I}^{*}, \beta^{*}\right\rangle \beta^{*}\right) .
$$

This implies that $\sigma_{0}$ acts on $V^{*}$ as the reflection in the hyperplane $L_{\beta *, 1}^{*}$ in $V^{*}$ and on $\sqrt{-}-1 V^{*}$ as the reflection in the hyperplane orthogonal to $\beta^{*}$.

The orbit of the set $\left\{\alpha_{1}^{*}, \cdots, \alpha_{n-1}^{*}\right\}$ under the action of the group generated by $\rho^{*}\left(\sigma_{1}\right), \cdots, \rho^{*}\left(\sigma_{n-1}\right)$ forms a root system $R^{*}$ of type $A_{n-1}$ in $V^{*}$ and $\left\{\alpha_{1}^{*}, \cdots, \alpha_{n-1}^{*}\right\}$ is a basis of $R^{*}$. The element $-\beta^{*} \in R^{*}$ is the highest root. Thus the generators $\rho^{*}\left(\sigma_{0}\right), \cdots, \rho^{*}\left(\sigma_{n-1}\right)$ of the image $\rho^{*}\left(\pi_{1}\left(S^{\prime}, t_{0}\right)\right)$ have the same relations as $w_{\imath}, 0 \leqq i \leqq n-1$. Therefore $\rho^{*}\left(\pi_{1}\left(S^{\prime}, t_{0}\right)\right)$ is isomorphic to the affine Weyl group of the root system of type $A_{n-1}$.

\section{§ 7. Period Mapping for the Fibration $\varphi: \mathfrak{X}^{\prime} \rightarrow S^{\prime}$}

In this final section, we shall define a period mapping for the family $\varphi: \mathfrak{X}^{\prime} \rightarrow S^{\prime}$. Let $\tilde{S}^{\prime}$ be the covering $\pi: \tilde{S}^{\prime} \rightarrow S^{\prime}$ of $S^{\prime}$ which is the quotient of the universal covering of $S^{\prime}$ by the kernel of the monodromy representation $\rho: \pi_{1}\left(S^{\prime}, t_{0}\right) \rightarrow$ Aut $\left(H_{2}\left(\mathfrak{X}_{t_{0}} \backslash \mathscr{D}_{t_{0}} ; \boldsymbol{Z}\right)\right)$. Thus $\tilde{S}^{\prime}$ is the regular covering of $S^{\prime}$ with the monodromy group $G=\rho\left(\pi_{1}\left(S^{\prime}, t_{0}\right)\right)$ as covering transformation group.

Let $t_{0} \cong S^{\prime}$ be the base point defined in Section 6 and write $\tilde{t}_{0}=\left(t_{0},[e]\right) \in \tilde{S}^{\prime}$, where $[e]$ is the unit of $\pi_{1}\left(S^{\prime}, t_{0}\right) / \operatorname{ker} \rho$. By the parallel displacement of a 2cycle $\gamma$ on $\dot{x}_{t_{0}} \backslash \mathscr{D}_{t_{0}}$ along paths in $S^{\prime}$ from $t_{0}$ to $t$, we have a horizontal family of homology classes $\gamma(\tilde{t}) \equiv H_{2}\left(\ddot{\mathfrak{x}}_{t} \backslash \mathfrak{D}_{t} ; Z\right),\left(\tilde{t} \boxminus \tilde{S}^{\prime}, t=\pi(\tilde{t})\right)$.

We associate a point $\tilde{t} \equiv \tilde{S}^{\prime}$ with an element $f_{\tilde{t}} \in \operatorname{Hom}_{\boldsymbol{Z}}\left(H_{2}\left(\mathfrak{X}_{t_{0}} \backslash \mathfrak{D}_{t_{0}} ; \boldsymbol{Z}, \boldsymbol{C}\right)\right.$ as follows: let

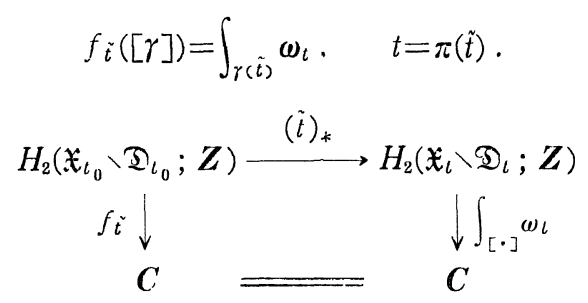

where $[\gamma]$ is the homology class of $\gamma$ and $(\tilde{t})_{*}$ is the mapping induced by the parallel displacement along the path from $t_{0}$ to $t$ which represents $\tilde{t}$. Thus we have a mapping

$$
\mathscr{Q}: \tilde{S}^{\prime} \longrightarrow \operatorname{Hom}_{\boldsymbol{Z}}\left(H_{2}\left(\mathfrak{X}_{t_{0}} \backslash \mathfrak{D}_{t_{0}} ; \boldsymbol{Z}\right), \boldsymbol{C}\right) .
$$

We call $\mathscr{P}$ the period mapping for $\varphi: \mathfrak{X}^{\prime} \rightarrow S^{\prime}$.

Let $\Omega$ be the affine subspace of Hom $\left(H_{2}\left(\mathfrak{X}_{t_{0}} \backslash \mathscr{D}_{t_{0}} ; \boldsymbol{Z}\right), \boldsymbol{C}\right)$ defined by (6.8). The fundamental group $\pi_{1}\left(S^{\prime}, t_{0}\right)$ acts on $\Omega$ as affine transformations as in Corollary 6.3. Let $L_{\alpha^{*, k}}^{*}$ be the hyperplane in $V^{*}$ defined by (6.9) and put

$$
\Omega^{\prime}=\Omega \backslash \bigcup_{\substack{\alpha \in \in R^{*} \\ k \in Z}}\left(\nu^{*}+L_{a \times, k}^{*}+\sqrt{-1} L_{\alpha *, 0}^{*}\right) .
$$


Theorem 7.1. The image of the period mapping $\mathscr{L}$ is in $\Omega^{\prime}$ and the mapping

$$
\mathscr{Q}: \widetilde{S}^{\prime} \longrightarrow \Omega^{\prime}
$$

is biholomorphic. The monodromy group $G$, the image of the monodromy representation $\rho: \pi_{1}\left(S^{\prime}, t_{0}\right) \rightarrow$ Aut $\left(H_{2}\left(\mathfrak{X}_{t_{0}} \backslash \mathfrak{D}_{t_{0}} ; Z\right)\right)$, acts on $\tilde{S}^{\prime}$ as covering transformation group and on $\Omega^{\prime}$ as affine transformations through the representation $\rho^{*}$. The period mapping $\mathscr{Q}$ is equivariant with these actions. Thus we have an isomorphism

$$
S^{\prime}=\tilde{S}^{\prime} / G \cong \Omega^{\prime} / G \text {. }
$$

Proof. As in the discussion of Section 6, an element of $S^{\prime}$ can be represented by an element $x \in \mathrm{t}+2 \pi \sqrt{-1} A \subset \mathrm{t}_{\boldsymbol{C}}^{\prime}$ and an element $w \in \widetilde{W}$ as follows. By Theorem 6.2, the monodromy group $G=\rho\left(\pi_{1}\left(S^{\prime}, t_{0}\right)\right)$ is isomorphic to the affine Weyl group $\widetilde{W}$ and $\mathrm{t}+2 \pi \sqrt{-1} A$ is a fundamental region of $\widetilde{W}$ in $t_{C}^{\prime}$. Thus, by (6.4), an element $\tilde{t} \subseteq \tilde{S}^{\prime}$ can be represented by an element $x_{\tilde{t}} \subseteq \mathrm{t}+$ $2 \pi \sqrt{-1} A$ and $w_{\tilde{t}} \Subset \widetilde{W}$ uniquely.

$$
\tilde{t}=\left(x_{\tilde{t}}, w_{\tilde{t}}\right), \quad x_{\tilde{t}} \in 1+2 \pi \sqrt{-1} A, \quad w_{\tilde{t}} \in \widetilde{W} .
$$

Let $x_{\tilde{t}}=\left(x_{1}, \cdots, x_{n}\right) \in \mathrm{t}+2 \pi \sqrt{-1} A$ and $\exp \left(x_{\tilde{t}}\right)=\left(t_{1}, \cdots, t_{n}\right)$, where $t_{\imath}=\exp \left(x_{\imath}\right)$. By (6.3),

$$
\arg t_{1}>\cdots>\arg t_{n}, \quad \arg t_{1}-\arg t_{n} \leqq 2 \pi, \quad \sum_{i=1}^{n} \arg t_{2}=0 .
$$

Let $U_{1}=\mathcal{U}_{1} \cap \mathfrak{E}_{t}$ (see (5.1)), $t=\pi(\check{t})$. Then

$$
U_{1} \cong\left\{\left(x_{1}, y_{1}\right)(\lambda: \mu): \boldsymbol{C}^{2} \times \boldsymbol{P}^{1} \mid \lambda y_{1}+\mu \prod_{i=1}^{n}\left(x_{1}-t_{2}\right)=0\right\} .
$$

Let $N(t)$ be the torus in $U_{1}$ defined as in Section 6 . Let $\tau_{\imath}(t)$ be the path from $\left(t_{\imath}, 0\right)(1: 0)$ to $\left(t_{\imath+1}, 0\right)(1: 0)$ in $U_{1}$ defined by

$$
\tau_{\iota}(t)(s)=\left(\left((1-s)\left|t_{i}\right|+s\left|t_{\imath+1}\right|\right) e^{\sqrt{-1}\left((1-s) \arg t_{\imath}+s \arg t_{\imath+1}\right)}, 0\right)(1: 0), \quad 0 \leqq s \leqq 1 .
$$

This path $\tau_{\imath}$ gives a 2-cycle $\Gamma_{\imath, \imath+1}(t)$ as in (6.7). Let $\nu(t)$ and $\alpha_{\imath}(t)$ be the homology classes of $N(t)$ and $\Gamma_{\imath, i+1}(t)$ respectively. Then these classes are the generators of $H_{2}\left(\mathfrak{X}_{t} \backslash \mathfrak{D}_{t} ; \boldsymbol{Z}\right)$ and the horizontal families of homology classes are given as follows:

$$
\begin{aligned}
& \nu(\tilde{t})=w_{\tilde{t}}^{-1}(\nu(t)), \\
& \alpha_{\imath}(\tilde{t})=w_{\tilde{t}}^{-1}\left(\alpha_{\imath}(t)\right),
\end{aligned}
$$

where the action of $\widetilde{W} \cong G$ on $H_{2}\left(\mathfrak{X}_{t} \backslash \mathfrak{D}_{t} ; \boldsymbol{Z}\right)$ is given by the same formula in Theorem 6.2.

Let $\nu, \alpha_{i}$ be the homology classes of 2-cycles $N, \Gamma_{\imath, \imath+1}$ on $\mathfrak{x}_{t_{0}} \backslash \mathscr{D}_{t_{0}}$ defined in Section 6. Then 


$$
\begin{aligned}
\mathscr{P}(\tilde{t})(\nu) & =\int_{\nu(\tilde{t})} \omega_{t} \\
& =\int_{w_{\tilde{t}}^{-1}(\nu(t))} \omega_{t} \\
& =\int_{\nu(t)} \omega_{t} \\
& =1 .
\end{aligned}
$$

Hence $\mathscr{P}(\tilde{t}) \in \Omega$.

$$
\begin{aligned}
\mathscr{P}(\tilde{t})\left(\alpha_{\imath}\right) & =\int_{a_{\imath}(\tilde{\imath})} \omega_{t} \\
& =\int_{w_{\tilde{t}}{ }^{1}\left(\alpha_{i}(t)\right)} \omega_{t} .
\end{aligned}
$$

If we write $w_{\tilde{t}}^{-1}\left(\alpha_{\imath}(t)\right)=c_{0} \nu(t)+\sum_{\imath=1}^{n-1} c_{\imath} \alpha_{\imath}(t)$, then

$$
\mathscr{L}(\tilde{t})\left(\alpha_{\imath}\right)=c_{0}+\sum_{i=1}^{n-1} c_{\imath} \int_{\alpha_{i}(t)} \omega_{t} .
$$

By the same arguement as in Section 4, we have

$$
\begin{aligned}
\int_{a_{\imath}(t)} \omega_{t} & =\frac{1}{2 \pi \sqrt{-1}}\left\{\log \left|\frac{t_{\imath+1}}{t_{\imath}}\right|+\sqrt{-1}\left(\arg \left(t_{\imath+1}\right)-\arg \left(t_{\imath}\right)\right)\right\} \\
& =\frac{1}{2 \pi}\left(\arg \left(t_{i+1}\right)-\arg \left(t_{i}\right)\right)-\frac{\sqrt{-1}}{2 \pi} \log \left|\frac{t_{i+1}}{t_{\imath}}\right| .
\end{aligned}
$$

Then we have

$$
\mathscr{P}(\tilde{t})\left(\alpha_{\imath}\right)=c_{0}+\sum_{i=1}^{n-1} c_{\imath}\left\{\frac{1}{2 \pi}\left(\arg \left(t_{\imath+1}\right)-\arg \left(t_{\imath}\right)\right)+\frac{\sqrt{-1}}{2 \pi} \log \left|\frac{t_{\imath}}{t_{\imath+1}}\right|\right\} .
$$

If $w_{i}=1$, then

$$
\mathscr{P}(\hat{t})\left(\alpha_{\imath}\right)=\frac{1}{2 \pi}\left(\arg \left(t_{\imath+1}\right)-\arg \left(t_{\imath}\right)\right)+\frac{\sqrt{-1}}{2 \pi} \log \left|\frac{t_{\imath}}{t_{\imath+1}}\right| .
$$

Let

$$
A^{*}=\left\{v^{*} \in V^{*} \mid v^{*}\left(\alpha_{2}\right)<0, v^{*}\left(\alpha_{1}+\cdots+\alpha_{n-1}\right)>-1\right\},
$$

then $\nu^{*}+A^{*}+\sqrt{-1} V^{*}$ is a fundamental region of $\rho^{*}\left(\pi_{1}\left(S^{\prime}, t_{0}\right)\right)$ in $\Omega^{\prime}$. Let

$$
x_{\tilde{t}}=\left(y_{1}, \cdots, y_{n}\right)+2 \pi \sqrt{-1}\left(z_{1}, \cdots, z_{n}\right) \quad\left(y_{1}\right):=\mathrm{t}, \quad\left(z_{i}\right) \in A .
$$

Then $t_{\imath}=e^{y_{i+2 \pi \sqrt{ }-1} z_{\imath}}$ and

$$
\begin{aligned}
\mathscr{P}(\tilde{t})\left(\alpha_{i}\right) & =\left(z_{\imath+1}-z_{i}\right)+\frac{\sqrt{-1}}{2 \pi} \log e^{y_{i}-y_{i+1}} \\
& =\left(z_{\imath+1}-z_{\imath}\right)+\frac{\sqrt{-1}}{2 \pi}\left(y_{\imath}-y_{\imath+1}\right) .
\end{aligned}
$$


Therefore it follows from (7.1) and (7.6) that $\mathscr{L}$ gives a bijective mapping from

to the space

$$
\left\{\tilde{t}=\left(x_{\tilde{t}}, 1\right) \boxminus \tilde{S}^{\prime} \mid x_{\tilde{t}} \in \mathrm{t}+2 \pi \sqrt{-1} A\right\}
$$

$$
\left\{f \equiv \Omega^{\prime} \mid f \in \nu^{*}+A^{*}+\sqrt{-1} V^{*}\right\} .
$$

By (7.2) and (7.3), the action of $\pi_{1}\left(S^{\prime}, t_{0}\right)$ on $\tilde{S}^{\prime}$ is compatible with that on $\Omega^{\prime}$. Therefore the period mapping $\mathscr{P}$ is bijective.

We next show that $\mathcal{P}$ is biholomorphic. Put

$$
x_{\imath}^{\prime}=\frac{1}{2 \pi}\left\{\left(\arg \left(t_{\imath+1}\right)-\arg \left(t_{\imath}\right)\right)+\sqrt{-1} \log \left|\frac{t_{\imath}}{t_{\imath+1}}\right|\right\} .
$$

Let $\left(a_{1}, \cdots, a_{n-1}\right)$ be a coordinate system of $S$ as in (5.1). By (7.5) and (7.6), it suffice to show that the Jacobian of the mapping $\left(a_{1}, \cdots, a_{n-1}\right) \rightarrow\left(x_{1}^{\prime}, \cdots, x_{n-1}^{\prime}\right)$ does not vanish, where

$$
x^{n}+a_{1} x^{n-1}+\cdots+a_{n-1} x+1==\prod_{i=1}^{n}\left(x-t_{2}\right) .
$$

Since

$$
e^{2 \pi \sqrt{v-1} x_{i}^{\prime}}=\frac{t_{i+1}}{t_{i}} \quad\left(\prod_{i=1}^{n} t_{i}=1\right),
$$

we shall calculate the Jacobians of the mappings

and

$$
\left(t_{1}, \cdots, t_{n-1}\right) \longrightarrow\left(t_{2} / t_{1}, \cdots, t_{n} / t_{n-1}\right)
$$

Since

$$
\left(t_{1}, \cdots, t_{n-1}\right) \longrightarrow\left(a_{1}, \cdots, a_{n-1}\right)
$$

$$
\begin{aligned}
& \frac{t_{n}}{t_{n-1}}=\frac{1}{t_{1} \cdots t_{n-2} t_{n-1}^{2}}, \\
& \frac{\partial\left(t_{n} / t_{n-1}\right)}{\partial t_{i}}= \begin{cases}-\frac{1}{t_{i}} \frac{1}{t_{1} \cdots t_{n-2} t_{n-1}^{2}}, & \text { if } i \leqq n-2, \\
-\frac{2}{t_{n-1}} \frac{1}{t_{1} \cdots t_{n-2} t_{n-1}^{2}} & \text { if } i=n-1,\end{cases}
\end{aligned}
$$

we have

$$
\begin{aligned}
J_{1} & =\frac{\partial\left(t_{2} / t_{1}, \cdots, t_{n} / t_{n-1}\right)}{\partial\left(t_{1}, \cdots, t_{n-1}\right)} \\
& =\frac{-1}{t_{1} \cdots t_{n-2} t_{n-1}^{2}}\left|\begin{array}{cccccc}
-t_{2} / t_{1}^{2} & 1 / t_{1} & 0 & & \cdots & 0 \\
0 & -t_{3} / t_{2}^{2} & 1 / t_{2} & 0 & \cdots & 0 \\
\vdots & & & \ddots & \\
0 & & 0 & -t_{n-1} / t_{n-2}^{2} & 1 / t_{n-2} \\
1 / t_{1} & & \cdots & 1 / t_{n-2} & 2 / t_{n-1}
\end{array}\right|
\end{aligned}
$$


From the $(n-1)$-th column to the second column, multiply the $i$-th column by $l_{\imath} / t_{\imath-1}$ and add it to the $(i-1)$-th column, then we have

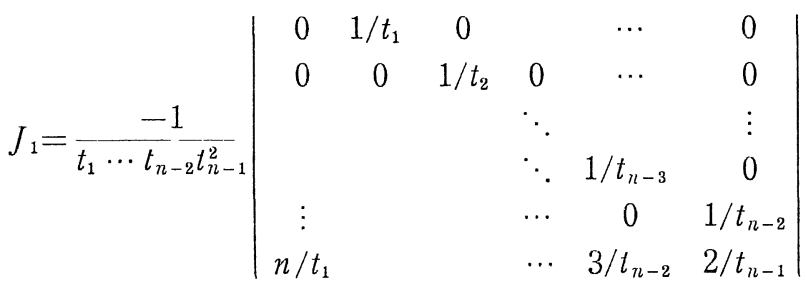

$$
\begin{aligned}
& =(-1)^{n-1} \frac{n}{t_{1}} \frac{1}{\left(t_{1} \cdots t_{n-1}\right)^{2}} \text {. }
\end{aligned}
$$

Thus $J_{1} \neq 0$. We next show that $J_{2}=\left(\partial\left(a_{1}, \cdots, a_{n-1}\right) / \partial\left(t_{1}, \cdots, t_{n-1}\right)\right) \neq 0$. Let $I_{\imath}$, $1 \leqq i \leqq n$, be the $i$-th elementary symmetric polynomial in variables $x_{1}, \cdots, x_{n}$. It is well known that

$$
\frac{\partial\left(I_{1}, \cdots, I_{n}\right)}{\partial\left(x_{1}, \cdots, x_{n}\right)}=\prod_{1 \leqq \imath<j \leqq n}\left(x_{\imath}-x_{\jmath}\right) .
$$

Thus the mapping $\left(x_{1}, \cdots, x_{n-1}\right) \rightarrow\left(I_{1}, \cdots, I_{n-1}\right)$ is locally biholomorphic on the submanifold defined by $x_{\imath} \neq x_{j}$ (if $i \neq j$ ) and $x_{1} \cdots x_{n}=1$, which is isomorphic to $S^{\prime}$. Therefore we have $J_{2} \neq 0$.

It follows from $J_{1} \neq 0, J_{2} \neq 0$, and (7.7) that the mapping $\left(a_{1}, \cdots, a_{n-1}\right) \rightarrow$ $\left(x_{1}^{\prime}, \cdots, x_{n-1}^{\prime}\right)$ is locally biholomorphic. It follows from the bijectivity of $\mathscr{Q}$ that $\mathscr{Q}$ is biholomorphic. The action of the monodromy group $G$ on $\widetilde{S}^{\prime}$ and $\Omega^{\prime}$ are equivariant and $G$ acts on these spaces freely. Therefore $\mathscr{L}$ induces an isomorphism

$$
S^{\prime} \cong \Omega^{\prime} / G
$$

and the theorem is proved.

\section{References}

[1] Barth, W., Peters, C. and Van de Ven, A., Compact Complex Surfaces, SpringerVerlag, Berlin Heidelberg New York Tokyo, 1984.

[2] Bourbaki, N., Groupes ct algèbres de Lie. Chap. 4, 5, 6, Hermann, Paris, 1968.

[3] Cayley, A., On the triple tangent planes of surfaces of the third order, Camb. and Dublin Math. Journal., IV (1849), 118-132; Collected papers I, Cambridge Univ. Press (1889), 445-456.

[4] Cartan, É., Sur la réduction ì sa forme canonique de la structure d'un groupe de transformations fini et continu, Amer. J. Math., 18 (1896), 1-61.

[5] Demazure, M., Surfaces de Del Pezzo II, III, IV, V, Séminaire sur Les Singularités des Surfaces, Lecture Notes in Math., 777 (1980), Springer-Verlag.

[6] Hartshorne, R., Algebraic Geometry, 3rd edition, Springer-Verlag, 1983.

[7] Looijenga, E., Rational surfaces with an anti-canonical cycle, Ann. of Math., 114 (1981), 267-322.

[8] Manin, Yu. 1., Cubic Forms, 2nd edition, North-Holland, Amsterdam, 1986. 
[9] Matsuzawa, J., Monoidal transformations of Hirzebruch surfaces and Weyl groups of type C, J. Fac. Sci. Univ. Tokyo, 35 (1988), 425-429; Correction, J. Fac. Sci. Univ. Tokyo, 36 (1989), 827.

[10] Naruki, I., Cross ratio variety as a moduli space of cubic surfaces, with Appendix by E. Looijenga, Proc. London Math. Soc. (3), 45 (1982), 1-30.

[11] Nguyễn Viêt Dũng, The fundamental groups of the spaces of regular orbits of the affine Weyl groups, Topology, 22 (1983), 425-435.

[12] Pinkham, H., Résolution simultanée de points doubles rationnels, Séminaire sur Les Singularités des Surfaces, Lecture Notes in Math., 777 (1980), Springer-Verlag. 